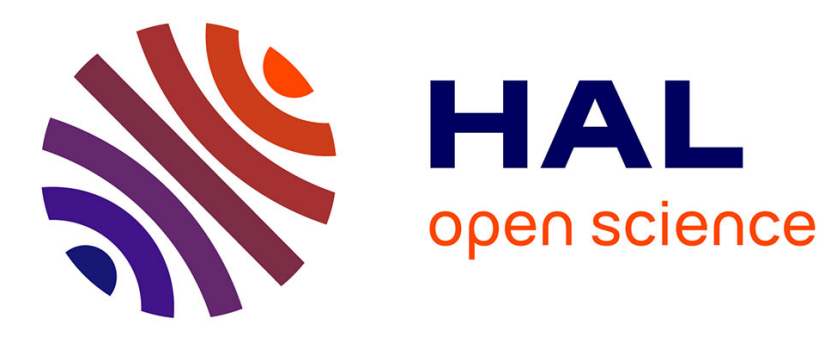

\title{
Absorbing Boundary Conditions for Solving N-Dimensional Stationary Schrödinger Equations with Unbounded Potentials and Nonlinearities
}

\author{
Pauline Klein, Xavier Antoine, Christophe Besse, Matthias Ehrhardt
}

\section{- To cite this version:}

Pauline Klein, Xavier Antoine, Christophe Besse, Matthias Ehrhardt. Absorbing Boundary Conditions for Solving N-Dimensional Stationary Schrödinger Equations with Unbounded Potentials and Nonlinearities. Communications in Computational Physics, 2011, 10 (5), pp.1280-1304. hal-00755605

\section{HAL Id: hal-00755605 \\ https://hal.science/hal-00755605}

Submitted on 23 Nov 2012

HAL is a multi-disciplinary open access archive for the deposit and dissemination of scientific research documents, whether they are published or not. The documents may come from teaching and research institutions in France or abroad, or from public or private research centers.
L'archive ouverte pluridisciplinaire HAL, est destinée au dépôt et à la diffusion de documents scientifiques de niveau recherche, publiés ou non, émanant des établissements d'enseignement et de recherche français ou étrangers, des laboratoires publics ou privés. 


\title{
Absorbing boundary conditions for solving $N$-dimensional stationary Schrödinger equations with unbounded potentials and nonlinearities
}

\author{
Pauline Klein ${ }^{\mathrm{a}, 1}$, Xavier Antoine $\mathrm{a}^{\mathrm{a}, 1, *}$, Christophe Besse ${ }^{\mathrm{b}, 1}$, Matthias Ehrhardt ${ }^{\mathrm{c}}$ \\ ${ }^{a}$ Institut Elie Cartan Nancy, Nancy-Université, CNRS UMR 7502, INRIA CORIDA Team, Boulevard des \\ Aiguillettes B.P. 239, 54506 Vandoeuvre-lès-Nancy, France \\ ${ }^{b}$ Laboratoire Paul Painlevé, CNRS UMR 8524, Simpaf Project Team - Inria CR Lille Nord Europe, \\ Université des Sciences et Technologies de Lille, Cité Scientifique, 59655 Villeneuve d'Ascq Cedex, France \\ ${ }^{c}$ Lehrstuhl für Angewandte Mathematik und Numerische Analysis, Fachbereich C-Mathematik und \\ Naturwissenschaften, Bergische Universität Wuppertal, Gaußstr. 20, 42119 Wuppertal, Germany
}

\begin{abstract}
We propose a hierarchy of novel absorbing boundary conditions for the one-dimensional stationary Schrödinger equation with general (linear and nonlinear) potential. The accuracy of the new absorbing boundary conditions is investigated numerically for the computation of energies and ground-states for linear and nonlinear Schrödinger equations. It turns out that these absorbing boundary conditions and their variants lead to a higher accuracy than the usual Dirichlet boundary condition. Finally, we give the extension of these ABCs to $N$-dimensional stationary Schrödinger equations.
\end{abstract}

Keywords: Absorbing boundary conditions, stationary Schrödinger equations, unbounded domain, spatially dependent potential, ground states computation

PACS: 03.65.-w, 42.82.Et, 43.30.Ma

2000 MSC: 35J10, 65M60, 65N30

\section{Introduction}

The solution of the Schrödinger equation occurs in many applications in physics, chemistry and engineering (e.g. quantum transport, condensed matter physics, quantum chemistry, optics, underwater acoustics, ....). The considered problem can appear in different forms: time-dependent or stationary equation, linear or nonlinear equation, inclusion of a variable potential among others. One of the main difficulty when solving the Schrödinger equation, and most particularly from a numerical point of view, is to impose suitable and physically admissible boundary conditions to solve numerically a bounded domain equation

${ }^{*}$ Corresponding author.

Email addresses: Pauline.Klein@iecn.u-nancy.fr (Pauline Klein),

Xavier.Antoine@iecn.u-nancy.fr (Xavier Antoine), Christophe.Besse@math.univ-lille1.fr

(Christophe Besse), ehrhardt@math.uni-wuppertal.de (Matthias Ehrhardt)

URL: http://www.iecn.u-nancy.fr/ antoine/ (Xavier Antoine),

http://math.univ-lille1.fr/ besse/ (Christophe Besse),

http://www-num.math.uni-wuppertal.de/ ehrhardt/ (Matthias Ehrhardt)

${ }^{1}$ Supported partially by the French ANR fundings under the project MicroWave NT09_460489 (http://microwave.math.cnrs.fr/). 
modeling an equation originally posed on an unbounded domain. Concerning the timedomain problem, many efforts have been achieved these last years. We refer the interested reader e.g. to the recent review paper [1] and the references therein for further details.

In this paper, we begin to focus on the solution to the one-dimensional stationary Schrödinger equation. For a given potential $V$ possibly extending to infinity, eventually nonlinear $(V:=V(x, \varphi)$, we want to solve the following equation

$$
\left(-\alpha \frac{d^{2}}{d x^{2}}+V\right) \varphi=E \varphi, \quad x \in \mathbb{R},
$$

or rewritten as

$$
\left(\frac{d^{2}}{d x^{2}}+\frac{1}{\alpha}[E-V]\right) \varphi=0, \quad x \in \mathbb{R},
$$

with some parameter $\alpha$ that allows for some flexibility. More precisely, we study the extension of the recently derived time-domain boundary conditions [2] to the computation of stationary states: we determine here the pair $(\varphi, E)$, for a given linear or nonlinear potential $V$. This eigenvalue problem is also known as the computation of ground states. The energy of the system is then the eigenvalue $E$ and the associated stationary state is the eigenfunction $\varphi$. In particular, we seek the fundamental stationary state which is linked to the smallest eigenvalue. In practice, higher order states are also of interest. After a careful numerical validation of the $1 \mathrm{D}$ case, we develop some $\mathrm{ABC}$ s for the generalized $N$-dimensional case

$$
-\alpha \Delta u+V(\mathbf{x}) u+f(u) u=E u
$$

in $\mathbb{R}^{N}$ for an unbounded potential $V$ and general nonlinearities. Another related problem which is not treated here is linear and nonlinear scattering. We refer to our extended version of the present paper [3] where a thorough study of scattering problems with the ABCs is developed.

Let us note here that numerical approaches based on solving the stationary Schrödinger with ABCs is not the only practical possibility. Indeed, other solutions may be used. The most widely used approach with applications for example in Bose-Einstein condensation is to bound the infinite space with a sufficiently large computational domain and then to impose a Dirichlet boundary condition on this fictitious boundary. The fact that we may fix the field at zero at the boundary is related to the property that ground states decay exponentially fast in the far-field. Then, spectrally accurate solutions can be efficiently obtained following the works by Bao et al. [4, 5]. Other solutions include whole space solutions based on spectral methods (see $[6,7]$ ). Let us also note the following other strategy $[8,9,10]$ for solving the linear Schrödinger equation with potential. If you approximate the potential one each subinterval by a constant, linear or quadratic function, then you get one each subinterval a problem that can be solved explicitly (harmonic oscillator, Airy equation) and these sub-problems are solved sequentially by some transfer algorithm. For constant potentials this approach was worked out in $[8,9,10]$ (see the MATLISE solver available at http://users.ugent.be/ vledoux/). Obviously, this strategy can be generalized to linear/quadratic potentials.

For the stationary Schrödinger equation (2), boundary conditions for solving linear scattering problems with a constant potential outside a finite domain have been proposed e.g. by Ben Abdallah, Degond and Markowich [11], by Arnold [12] for a fully discrete Schrödinger equation and in a two-dimensional quantum waveguide by Lent and Kirkner [13, 14]. Other 
recent contributions include $[15,16]$ where the case of finite range potentials is treated. The case of bound states can be found for specific one-dimensional linear Schrödinger equations in $[17,18,19,20,21]$. These boundary conditions are needed e.g. to improve existing simulation tools for semiconductors that allows to investigate certain stationary (and also transient) behavior of the devices, like conductance, capacity, current-voltage curves. Often the physical relevant effects take place only in a small subregion of the device, and the novel absorbing boundary conditions offer the possibility to confine the computations to this small domain. We refer the reader to $[22,23,24,25]$ for more application details.

The goal of this work is to propose and numerically validate some new boundary conditions for modeling linear and nonlinear variable unbounded potentials stationary onedimensional Schrödinger equations with application to ground-state computation. Finally, we extend these absorbing boundary conditions to higher dimensional problems. The paper is organized as follows. In Section 2, we explain how to obtain the stationary boundary conditions from the time-dependent case. Sections 3 and 4 are respectively devoted to their applications to linear and nonlinear eigenstate computation. Section 5 gives an extension of the boundary conditions to the $N$-dimensional space for nonlinear stationary Schrödinger equations with unbounded potentials. Finally, Section 6 draws a conclusion and give an outlook for possible future research directions.

\section{Absorbing boundary conditions: from the time-domain to the stationary case: the one-dimensional case}

In order to derive some absorbing boundary conditions ( $A B C s$ ) for the stationary Schrödinger equation (2), let us first start with the time-domain situation. In case of the timedependent Schrödinger equation with a linear or nonlinear potential $\widetilde{V}$

$$
\begin{cases}i \partial_{t} u+\partial_{x}^{2} u+\widetilde{V} u=0, & \forall(x, t) \in \mathbb{R} \times \mathbb{R}^{+}, \\ u(x, 0)=u_{0}(x), & x \in \mathbb{R},\end{cases}
$$

the following second- and fourth-order $A B C s$ on the boundary $\Sigma \times \mathbb{R}^{+}$

$$
\begin{aligned}
\mathrm{ABC}_{2}^{2}: & \partial_{\mathbf{n}} u=i \sqrt{i \partial_{t}+\widetilde{V}} u \\
\mathrm{ABC}_{2}^{4}: & \partial_{\mathbf{n}} u=i \sqrt{i \partial_{t}+\widetilde{V}} u-\frac{\partial_{\mathbf{n}} \widetilde{V}}{4}\left(i \partial_{t}+\widetilde{V}\right)^{-1} u,
\end{aligned}
$$

were derived recently in [2]. Here, for an operator $A, \sqrt{A}$ denotes the square-root operator of $A[26]$ with respect to its spectral decomposition. The fictitious boundary $\Sigma$ is given by the two interval endpoints $x_{\ell}$ and $x_{r}$. The outwardly directed unit normal vector to the bounded computational domain $\Omega=] x_{\ell} ; x_{r}$ [ is denoted by $\mathbf{n}$.

To obtain some ABCs for the stationary equations (1) or (2), we consider these equations

supplied with a new potential: $\widetilde{V}:=-V / \alpha$. Moreover, we are seeking some time-harmonic solutions $u(x, t):=\varphi(x) e^{-i \frac{E}{\alpha} t}$. Since

$$
i \partial_{t} u=\frac{E}{\alpha} \varphi(x) e^{-i \frac{E}{\alpha} t}
$$

we have

$$
\sqrt{i \partial_{t}+\widetilde{V}} u=\frac{1}{\sqrt{\alpha}} \sqrt{E-V}\left(\varphi e^{-i \frac{E}{\alpha} t}\right)
$$


These considerations yield some stationary $A B C$ s that we designate by $\mathrm{SABC}^{M}$ ('S' stands for stationary and $M$ denotes the order of the boundary condition) :

$$
\begin{array}{ll}
\mathrm{SABC}^{2}: & \partial_{\mathbf{n}} \varphi=i \frac{1}{\sqrt{\alpha}} \sqrt{E-V} \varphi, \text { on } \Sigma, \\
\mathrm{SABC}^{4}: & \partial_{\mathbf{n}} \varphi=i \frac{1}{\sqrt{\alpha}} \sqrt{E-V} \varphi+\frac{1}{4} \frac{\partial_{\mathbf{n}} V}{E-V} \varphi, \quad \text { on } \Sigma .
\end{array}
$$

The second- and fourth-order ABCs for the time-dependent Schrödinger equation (4), (5) were developed under a high frequency assumption [2]. This relation can be translated to the stationary case in terms of links between $E$ and $V$. The new relations will be given for the eigenvalues problems in the next dedicated sections.

Remark 1. For the time-dependent case [2], we constructed two families of ABCs, denoted by $\mathrm{ABC}_{1}^{M}$ and $\mathrm{ABC}_{2}^{M}$. These $\mathrm{ABC}$ all coincide if the potential is time-independent. In the stationary case, all the potentials fall into this category and thus the ABCs are equivalent. Hence, we get the unique class of stationary $\mathrm{ABCs}, \mathrm{SABC}^{M}$ (without subscript index). For convenience, the form of the boundary conditions (6)-(7) is based on $\mathrm{ABC}_{2}^{M}$ (we refer to [2] for more technical details).

\section{Application to the computation of stationary states: the linear case}

Let us consider the Hamiltonian $H$

$$
H=-\alpha \frac{d^{2}}{d x^{2}}+V(x), \quad x \in \mathbb{R}
$$

defined through $\alpha$ and $V$. The task here is to determine the pair $\left(\phi_{E}, E\right)$ solution to the eigenvalue problem:

$$
H \phi_{E}=E \phi_{E}, \quad x \in \mathbb{R} .
$$

This problem can also be formulated as follows: find the eigenvalues $\left(E_{n}\right)_{n \in \mathbb{N}}$ (energies) and the associated real-valued eigenfunctions $\left(\phi_{n}\right)_{n \in \mathbb{N}}$ (eigenstates or ground states) as solutions of: $H \phi_{n}=E_{n} \phi_{n}, x \in \mathbb{R}$. To fix the eigenfunction, it is necessary to impose a normalization condition: $\left\|\phi_{E}\right\|_{L^{2}(\mathbb{R})}=1$. Let us begin with the case where the potential does not depend on the eigenfunction (called linear case here). The nonlinear case will be treated later in Section 4.

\subsection{Square-root $A B C s$}

Before discussing the difficulties related to the ABCs, let us consider the numerical solution of our problem with a homogeneous Dirichlet boundary condition. The variational formulation of (9) reads

$$
-\alpha\left[\partial_{\mathbf{n}} \phi_{E} \psi\right]_{x_{\ell}}^{x_{r}}+\alpha \int_{\Omega} \partial_{x} \phi_{E} \partial_{x} \psi d x+\int_{\Omega} V \phi_{E} \psi d x=E \int_{\Omega} \phi_{E} \psi d x
$$

for some test-functions $\psi \in H_{0}^{1}(\Omega)$ [27]. Let $\mathbb{S}^{0}, \mathbb{M}^{0}$ and $\mathbb{M}_{V}^{0}$ be respectively the stiffness matrix, mass and generalized mass matrices associated with the potential $V$ for $\mathbb{P}_{1}$ finite element and a homogeneous Dirichlet boundary condition (these matrices are some elements 
of $\left.\mathcal{M}_{n_{h}-1}(\mathbb{R})\right)$. The discrete problem can be classically formulated as the following generalized eigenvalue problem: find the pair $\left(E, \phi_{E}\right)$ as solution to

$$
\left\{\begin{array}{l}
\left(\alpha \mathbb{S}^{0}+\mathbb{M}_{V}^{0}\right) \boldsymbol{\phi}_{E}=E \mathbb{M}^{0} \boldsymbol{\phi}_{E} \\
\left\|\mathbb{M}^{0} \boldsymbol{\phi}_{E}\right\|_{2}=1
\end{array}\right.
$$

which is a generalized eigenvalue problem with an equality constraint. Here, $\phi_{E}$ is a vector in $\mathbb{R}^{n_{h}-1}$ which is normalized by: $\left\|\mathbb{M}^{0} \phi_{E}\right\|_{2}=1\left(\|\cdot\|_{2}\right.$ being the usual Euclidian norm in $\mathbb{R}^{n_{h}-1}$ ). The global algorithm complexity is essentially the sum of the complexities for building the sparse finite element matrices and for computing the eigenvalue problem. In this paper, we use Matlab's eigs function which provides the $p$ smallest positive eigenvalues corresponding to the generalized eigenvalue problem. This function automatically normalizes the eigenvectors in the Euclidian norm hence fulfilling the normalization constraint in (11). eigs is associated with the software ARPACK. In the case where the potential is not always positive, we use the property that the smallest eigenvalue $E_{0}$ is larger than the minimum of the potential $V_{\min }$ and solve (11) by a translation of $-V_{\min }$. Finally, the solution to (11) generates the sequence of the $p$ first eigenvalues $\left(E_{n}^{0}\right)_{0 \leq n \leq p-1}$, eigenvectors $\left(\phi_{n}^{0}\right)_{0 \leq n \leq p-1}$ and finite element eigenfunctions $\left(\phi_{n}^{0}\right)_{0 \leq n \leq p-1}$ associated with the Dirichlet boundary condition. Since this eigenvalue problem is linear with respect to $E$, we can solve it without using e.g. a fixed point algorithm, unlike the case of including a square-root $\mathrm{ABC}$ as it is explained below. For this reason, the solution is called "direct" in the sequel of the paper.

Let us consider now the $\mathrm{SABC}^{2}$ boundary condition

$$
\partial_{\mathbf{n}} \phi_{E}=\frac{i}{\sqrt{\alpha}} \sqrt{E-V} \phi_{E}, \quad \text { on } \Sigma .
$$

The main difficulty with this boundary condition is its nonlinear dependence on $E$. As a consequence, we cannot isolate the terms $\left(E, \phi_{E}\right)$ in the right-hand side of $(10)$ in a linear way, that is under the form $E \phi_{E}$. More precisely, the nonlinear eigenvalue problem to solve is

$$
\left\{\begin{array}{l}
\left(\alpha \mathbb{S}+\mathbb{M}_{V}+\mathbb{B}_{M}\left(E^{M}\right)\right) \phi^{M}=E^{M} \mathbb{M} \phi^{M} \\
\left\|\mathbb{M} \phi^{M}\right\|_{2}=1
\end{array}\right.
$$

using the matrix notations of the scattering problem. We precise that both the eigenvalues and eigenfunctions depend on the chosen boundary condition $\mathrm{SABC}^{M}$ by the notation: $\left(E^{M}, \phi^{M}\right)$. The first $p$ eigencomponents are indexed as follows: $\left(E_{n}^{M}, \phi_{n}^{M}\right)$, with $0 \leq n \leq p-1$. The nonlinear dependence on the boundary term is given by the presence of $\mathbb{B}_{M}\left(E^{M}\right)$. To solve the eigenvalue problem with $\mathrm{SABC}^{M}$, we have to apply an iterative scheme like a fixed point method (with a prescribed tolerance $\epsilon$ ) and update $E^{M}$ at each iteration step $j$.

This procedure implies that we have to a priori choose an eigenvalue of index $n$ (denoted by $E_{n}^{M}$ ) that we wish to calculate. This is an important drawback since we have to a priori compute successively all the eigenvalues and associated eigenvectors. In fact, it appears that eigs is also able to provide an approximation of the first $p$ eigenvalues $\left(E_{n}^{M, j}\right)_{0 \leq n \leq p-1}$ of $\left(E_{n}^{M}\right)_{0 \leq n \leq p-1}$ and the corresponding eigenvectors $\phi_{n}^{M}$. As a consequence, we also have to recompute the boundary terms arising in $\mathbb{B}_{M}\left(E_{n}^{M, j}\right)$. Hence, the fixed point algorithm reads

$$
\left\{\begin{aligned}
& \left(\alpha \mathbb{S}+\mathbb{M}_{V}+\mathbb{B}_{M}\left(E_{n}^{M, j}\right)\right) \phi^{M, j+1}=E^{M, j+1} \mathbb{M} \phi^{M, j+1}, \\
& \left\|\mathbb{M} \phi^{M, j+1}\right\|_{2}=1
\end{aligned}\right.
$$


each linear problem being solved by using the Matlab routine eigs. More generally, for a boundary condition with a nonlinear dependence on the energy $E$, we use an associated fixed point algorithm. Even if we iterate through a fixed point algorithm, it appears that the algorithm also simultaneously gives some approximations of the other eigenvalues and eigenvectors (see the numerical section). This approach is therefore designated by "direct" if we only iterate on one a priori fixed eigenvalue. This algorithm can be applied successively by iteration using the fixed point algorithm and keeping only the computed eigenvalue and eigenvector related to the current iteration. Of course, the resulting algorithm is more expensive but at the same time more accurate. This approach is designated by "loop" in the sequel. Let us remark that there is no difference between both approaches for the Dirichlet problem.

\subsection{Linearized $A B C s$}

Unlike the case of the Dirichlet problem, we previously saw that the algorithm related to the square-root $\mathrm{ABCs}$ is iterative because of the nonlinearity. To avoid this problem, we can linearize $\mathrm{SABC}^{2}$ and $\mathrm{SABC}^{4}$. The principle is based on a Taylor's expansion in the regime $E \ll V$. This asymptotic regime is justified in particular for an harmonic potential $V(x)=\frac{1}{2} x^{2}$ since $V$ grows quickly as soon as we do not place the boundary too close to the origin and we restrict our computations to relatively not too high energies. For the boundary condition $\mathrm{SABC}^{2}(12)$, this leads to the approximation of $\mathrm{SABC}^{2}$ by $\mathrm{SABC}_{\text {lin }}^{2}$ given by

$$
\partial_{\mathbf{n}} \tilde{\phi}_{E}=-\frac{\sqrt{V_{\ell, r}}}{\sqrt{\alpha}} \tilde{\phi}_{E}+\frac{1}{2} \frac{E}{\sqrt{\alpha} \sqrt{V_{\ell, r}}} \tilde{\phi}_{E} .
$$

Next we can isolate the linear part according to $E$ as $\partial_{\mathbf{n}} \tilde{\phi}_{E}=\beta_{\ell, r}^{2} \tilde{\phi}_{E}+E \gamma_{\ell, r}^{2} \tilde{\phi}_{E}$, with $\beta_{\ell, r}^{2}$ and $\gamma_{\ell, r}^{2}$ defined by (15). Including these ABCs in the weak formulation (10) leads, after discretization by the $\mathbb{P}_{1}$ finite element method, to the following linear eigenvalue problem $(M=2)$

$$
\left\{\begin{array}{l}
\left(\alpha \mathbb{S}+\mathbb{M}_{V}+\mathbb{C}_{M}\right) \tilde{\boldsymbol{\phi}}^{M}=\tilde{E}^{M}\left(\mathbb{M}+\mathbb{D}_{M}\right) \tilde{\boldsymbol{\phi}}^{M} \\
\left\|\mathbb{M} \tilde{\boldsymbol{\phi}}^{M}\right\|_{2}=1
\end{array}\right.
$$

We have defined the two matrices $(M=2)$

$$
\mathbb{C}_{M}=\left(\begin{array}{cccc}
\alpha \beta_{\ell}^{M} & 0 & 0 & 0 \\
0 & \ldots & \ldots & 0 \\
0 & \ldots & \cdots & 0 \\
0 & \cdots & \cdots & 0 \\
0 & 0 & 0 & \alpha \beta_{r}^{M}
\end{array}\right), \mathbb{B}_{M}=\left(\begin{array}{cccc}
-\alpha \gamma_{\ell}^{M} & 0 & 0 & 0 \\
0 & \ldots & \ldots & 0 \\
0 & \ldots & \ldots & 0 \\
0 & \ldots & \ldots & 0 \\
0 & 0 & 0 & -\alpha \gamma_{r}^{M}
\end{array}\right) .
$$

Problem (16) is directly solved without iteration by using eigs. The computational cost is therefore the same as for a Dirichlet boundary condition. Furthermore, since we do not have to iterate, the algorithm provides simultaneously the first $p$ eigenvalues $\left(\tilde{E}_{n}^{M}\right)_{0 \leq n \leq p-1}$ and associated eigenvectors $\left(\tilde{\boldsymbol{\phi}}_{n}^{M}\right)_{0 \leq n \leq p-1}$. The resulting algorithm is called direct. In the case of $\mathrm{SABC}^{4}$, a similar strategy of linearization of (7) leads to the approximation

$$
\partial_{\mathbf{n}} \tilde{\phi}_{E}=\beta_{\ell, r}^{4} \tilde{\phi}_{E}+E \gamma_{\ell, r}^{4} \tilde{\phi}_{E}
$$


with $\beta_{\ell, r}^{4}$ and $\gamma_{\ell, r}^{4}$ respectively given by

$$
\beta_{\ell, r}^{4}=\beta_{\ell, r}^{2}-\frac{1}{4} \frac{\partial_{\mathbf{n}} V_{\mid x=x_{\ell, r}}}{V_{\ell, r}}, \gamma_{\ell, r}^{4}=\gamma_{\ell, r}^{2}-\frac{1}{4} \frac{\partial_{\mathbf{n}} V_{\mid x=x_{\ell, r}}}{V_{\ell, r}^{2}}
$$

by using the approximation

$$
\frac{1}{4} \frac{\partial_{\mathbf{n}} V}{E-V} \approx-\frac{1}{4} \frac{\partial_{\mathbf{n}} V}{V}-\frac{1}{4} \frac{\partial_{\mathbf{n}} V}{V^{2}} E
$$

Adapting the functions, our problem can be written as (16).

\subsection{Numerical examples}

Example 1 (Harmonic potential). We first consider the well-known (positive) harmonic potential $V(x)=\frac{1}{2} x^{2}$, i.e. the equation to solve is

$$
-\frac{1}{2} \phi_{E}^{\prime \prime}+\frac{1}{2} x^{2} \phi_{E}=E \phi_{E}, \quad x \in \mathbb{R}
$$

with $\alpha=\frac{1}{2}$. The square-integrable normalized solutions of (21) are the Hermite functions

$$
\phi_{n}^{\mathrm{ex}}(x)=\frac{\pi^{-1 / 4}}{\sqrt{2^{n} n !}} e^{x^{2} / 2} \frac{d^{n}}{d x^{n}}\left(e^{-x^{2}}\right), \quad n \geq 0
$$

and the corresponding eigenvalues (energies) are: $E_{n}^{\mathrm{ex}}=n+\frac{1}{2}$. The eigenfunctions $\phi_{n}^{\mathrm{ex}}(x)$ vanish for $|x| \rightarrow \infty$, but this decay is slower and slower as $n$ grows.

Let us recall that, for the case of the square-root ABCs, we have the direct and loop strategies. In the sequel, when we present an error calculation with respect to $x_{r}$ or $h$, this is clearly obtained by the direct approach since $n$ is fixed. When we compute a range of eigenvalues (curves with $n$ as abscissa), we report the results for both strategies to compare the respective accuracies.

A first numerical test consists in presenting the error on both the energy and eigenfunctions depending on the computational domain size. For the harmonic potential, we always consider a symmetric domain $\Omega=]-x_{r} ; x_{r}$. For a fixed $n$, the value of an eigenfunction is closer to zero as $x_{r}$ becomes larger. This means that we should observe the impact of the ABCs compared to the homogeneous Dirichlet boundary condition depending on the location of $x_{r}$. Figure 1 reports, for the fundamental state $n=0$ and in logarithmic scale, the absolute error on the eigenvalue $|\Delta E|=\left|E_{n}^{\text {num }}-E_{n}^{\text {ex }}\right|$ and the error in the $L^{2}$-norm of the eigenfunction $\|\Delta \phi\|_{L^{2}(\Omega)}=\left\|\phi_{n}^{\text {num }}-\phi_{n}^{\text {ex }}\right\|_{L^{2}(\Omega)}$ when the right endpoint $x_{r}$ varies between 1 and 7 .

Figure 2 presents similar results for $n=4$ and $x_{r}$ varying between 3 and 10 . The calculations are obtained for the numerical eigenvalues $E_{n}^{\text {num }}$ equal to $E_{n}^{M}$ (for $\mathrm{SABC}^{M}$ ) or $\tilde{E}_{n}^{M}$ (for $\mathrm{SABC}_{\text {lin }}^{M}$ ), depending on the order $M$ of the $\mathrm{ABC}$ and its type (square-root or linearized). In the nonlinear case, corresponding to $\mathrm{SABC}^{M}$, the number of iterations is 50 to reach convergence with $\varepsilon=10^{-12}$. The spatial step size is $h=1 \cdot 10^{-3}$.

For $n$ fixed, we observe an accuracy improvement for both boundary conditions when $x_{r}$ grows. When $x_{r}$ is close to the origin (for example $x_{r}=1$ for $n=0, x_{r}<3$ for $n=4$ ), all the conditions lead to inaccurate results. However, even for these small values of $x_{r}$, the linearized ABCs already give an approximation of the eigenvalue while this is not the case 
for the Dirichlet boundary condition as well as for $\mathrm{SABC}^{2,4}$. Indeed for $x_{r}=3$ and $n=4$, the $\mathrm{ABCs} \mathrm{SABC}_{\text {lin }}^{2,4}$ give $\tilde{E}_{n}$ with an error equal to $10^{-2}$ when the error for the homogeneous Dirichlet boundary condition is about $10^{-1}$ and 1 for the square-root ABCs. The same remark holds for $n=0$.

It seems from these tests that the linearized ABCs are the most robust boundary conditions concerning the size of the computational domain. From a general point of view, the ABCs always provide a better precision, at least about the same as with the Dirichlet boundary condition but often far better. The ABCs of different orders generally give a similar accuracy with however a better accuracy behaviour of the square-root ABCs but at a higher computational cost. They improve the accuracy of the Dirichlet boundary condition from a factor between 10 and $10^{3}$ according to the configuration, before attaining the saturation zone. After a certain value of $x_{r}$, all the boundary conditions lead to the same accuracy which only depends on the spatial mesh size. For the computation of the eigenfunctions, this value can be estimated to $x_{r}=6$ for $n=0$ and to $x_{r}=6.5$ for $n=4$.

We also remark that we must increase $x_{r}$ as $n$ grows to get the same accuracy. To confirm this, we compute the variation of the error when $\Omega=]-x_{r} ; x_{r}$ [ is fixed and $n$ varies. We set $x_{r}=4$ and for $n \in[0,10]$ we report the error on the eigenvalues $E_{n}$ (Figure 3 ) for both the "direct" and "loop" approaches.

For all the boundary conditions, we can clearly see that the accuracy decays as $n$ increases. Indeed, the $\mathrm{ABCs}$ have been built in the high frequency regime. In our context, this means that we require that:

$$
E_{n}-V_{r} \ll 0
$$

holds for a given point $x_{r}$ and for a fixed potential $V$. As a consequence, this limits the calculation of energies under the condition $E_{n} \ll x_{r}^{2} / 2$ for example in the harmonic case. In the proposed simulation, setting $x_{r}=4$ leads to $E_{n} \ll 8$, which is coherent with the observations in Figure 3. Another way to interpret this property is that increasing the accuracy and the range of eigenvalues must be a priori guided by relation (23). To visualize this, we show in Figure 4 the potential $V$ as well as the first energies $E_{n}$. We can read from this figure the abscissa $x$ where $E-V$ becomes negative and we can have a first idea of the choice of the minimal abscissa $x_{r}$ to choose to get a sufficiently large gap between $E$ and $V\left(x_{r}\right)$ according to $(23)$.

For example, for the fundamental state $n=0$, the energy associated with $E_{0}$ is the lowest level red curve. From the intersection with the curve of $V(x)$, we can see that $E_{0}-V(x)$ is negative for $x \geq 1$ and we can estimate that the difference between $E_{0}$ and $V(x)$ will be enough starting from about $x \geq 2$. Coming back to Figure 1 confirms these values since choosing $x_{r}=1$ provides a possible computation but does not necessarily converge towards $E_{0}$ while setting $x_{r}=2$ gives a correct approximation of $E_{0}$. We can do the same analysis for $n=4$ (fifth red curve from the bottom). We see that $E_{4}-V(x)$ is negative from $x \geq 3$ and "very negative" after $x \geq 4$. These values must be connected with the curves of Figure 2 .

On this example $(n=4)$, we also remark that the linearized ABCs are more accurate than the original square-root $\mathrm{ABCs}$, with a gain of a factor 10 in precision. This remark could also have been made on Figure 2 corresponding to $n=4$, most particularly for the computation of the eigenvalue. The precision obtained for $x_{r} \geq 4.5$ with the linearized boundary conditions is the same as for the square-root boundary conditions but the linearization yields an accuracy improvement on smaller computational domains while the iterative algorithm for the squareroot conditions does not converge $\left(2 \leq x_{r} \leq 3\right)$. Moreover, let us note that the spectrum is simultaneously obtained in the linear case without iterating which is a crucial gain compared 
to the "loop" approach, showing hence the need of linearizing. As a consequence, the ABCs $\mathrm{SABC}_{\text {lin }}^{M}$ are, for a similar computational cost, to privilege to the Dirichlet boundary condition for accuracy purpose and/or for reducing the computational domain. Let us also finally remark that the gain in terms of accuracy of the "loop" approach is interesting as we can see it in Figure 2(a) but for a relatively higher computational complexity.

We now wish to compare the performances of the linearized and square-root ABCs. The previous curves illustrated the question of accuracy. Generally speaking, the square-root ABCs provide a better accuracy but at a higher computational cost even for the "direct" approach since a fixed point is required. We show in Figure 5 the number of iterations when using $\mathrm{SABC}^{2}$ and $\mathrm{SABC}^{4}$, with respect to $x_{r}$, for two situations: $n=0$ and $n=4$. Figures 5(a) and 5(b) must be connected to Figures 1 and 2 which are their equivalent in terms of accuracy.

For the first value of $x_{r}$, we often observe the divergence of the algorithm (the maximal number of iterations of the fixed point algorithm is 20). Again, this is one of the interesting property of the linear ABCs since, if we go back to Figures 1 and 2, they also give a rough estimate of the eigenvalue.

For a slightly larger value of $x_{r}$, the number of iterations stagnates to 5 . Finally, when the maximal accuracy is reached, the algorithm needs 2 or 3 iterations. Globally, the computational costs for the square-root ABCs are roughly 5 times the costs for the linear ones and the Dirichlet boundary condition. At the same time, a higher accuracy is obtained by the square-root ABCs.

Finally, we present in Figures $6(n=0)$ and $7(n=4)$ the influence of the discretization on the accuracy for a given computational domain ] $-x_{r} ; x_{r}\left[\right.$. We fix $x_{r}$ and report the errors $|\Delta E|$ and $\|\Delta \phi\|_{L^{2}(\Omega)}$ depending to the mesh size $h$, for $h$ between $h=5 \cdot 10^{-2}$ and $h=1 \cdot 10^{-4}$. The value of $x_{r}$ is chosen such that the saturation of the error has not been reached yet so that we can see an effect of the ABCs compared to the Dirichlet boundary condition.

One remarkable property is that for $n=0$ (Figure 6) the accuracy remains increasing with the ABCs by refining the mesh while this is not the case for the Dirichlet boundary condition. Indeed, we cannot gain more accuracy after $h=10^{-2}$ if we do not increase the size of the computational domain. Concerning the ABCs (which are already more accurate than the Dirichlet boundary condition for $h=10^{-2}$ ), we can still improve the solution by refining, most particularly with $\mathrm{SABC}^{4}$. Note that this remark holds for both the eigenvalues and eigenvectors.

Example 2 (Pöschl-Teller potential). The potential that we analyze now has the property to lead to negative eigenvalues. A necessary condition to justify the application of the previous approach is that

$$
V\left(x_{r}\right)-E \geq 0 .
$$

Hence, according to $n$ and the rank of the eigenvalue that we are looking for, we have to choose $x_{r}$ sufficiently large so that condition (24) is fulfilled. Since $V$ is negative and even if we have $E \ll V$, then linearizing $\mathrm{SABC}^{2,4}$ by using a Taylor's expansion with respect to $E / V$ is no longer relevant since $V$ can be equal to zero. Let us set $V_{\min }=\min _{x \in \mathbb{R}} V(x)$ and using the property that the Schrödinger equation is linear, we define a new positive potential $W=V-V_{\min }$ and $F_{n}=E_{n}-V_{\min }$. Problem (8)-(9) is then equivalent to

$$
-\alpha \phi_{E}^{\prime \prime}+W \phi_{E}=F_{n} \phi_{E} .
$$


The boundary conditions $\mathrm{SABC}_{\text {lin }}^{2,4}$ are so the linearized versions of $\mathrm{SABC}^{2,4}$ according to $1 /\left(V-V_{\min }\right)$ (and not $\left.1 / V\right)$ by using the equivalent assumption: $E-V_{\min } \ll V-V_{\min }$.

The Pöschl-Teller potential [28] is given by

$$
V(x)=-\frac{\lambda(\lambda+1)}{\cosh ^{2}(x)},
$$

and $\alpha=1 \mathrm{in}(8)$. This potential is always negative (see Figure $8(\mathrm{a})$ ). For $\lambda=9$, it leads to nine eigenvalues: $E_{n}=-(9-n)^{2}, \quad 0 \leq n \leq 8$. In Figure 8(b), we plot the different energy levels, compared to the potential. To take into account the translation, we rather present $V(x)-V_{\min }$ and $E_{n}-V_{\min }$.

For a given eigenstate, we can a priori estimate the size of the computational domain to consider that the high frequency hypothesis is satisfied and that the convergence of the iterative algorithm occurs. We set $h=5 \cdot 10^{-4}$ and analyze, for $n$ fixed, $0 \leq n \leq 8$, the error on $E_{n}$ for the different ABCs depending on the position of $x_{r}$. We depict the results in Figure 9 for $n=0, n=4$ and $n=8$. The ABCs always improve the accuracy compared to the Dirichlet boundary condition. This is most particularly clear for large $n$. For $n=8$ and ] $-5 ; 5$ [, the accuracy obtained with the Dirichlet boundary condition is less than $10^{-2}$ and about $5 \cdot 10^{-5}$ for $\mathrm{SABC}^{2,4}$. To get a similar precision with the Dirichlet boundary condition, we would have to choose $x_{r}=10$ leading therefore to a significant larger computational domain. The effect of the linearized ABCs is variable. For $n=0$ the $\mathrm{ABCs} \mathrm{SABC}_{\text {lin }}^{2,4}$ are almost as precise as the $\mathrm{ABCs} \mathrm{SABC}^{2,4}$, but when $n$ increases, the accuracy is similar to the one obtained by using the Dirichlet boundary condition.

For the same potential, we observe in Figure 10 the error on $E_{n}$ for $x_{r}$ fixed. For $x_{r}=2$, we notice a factor 10 to 100 between the Dirichlet boundary condition and the ABCs for the states $2 \leq n \leq 6$. The second- and fourth-order ABCs have a similar accuracy. At $x_{r}=4$, all the boundary conditions are equivalent for the first eigenstates but when $n$ grows the ABCs remain accurate while the Dirichlet boundary condition is less precise $(n=6, n=7)$. Indeed, they yield an accuracy of the eigenvalue about $10^{-3}$ while the Dirichlet boundary condition gives only $10^{-1}(n=8)$.

Remark 2. In [3, 29], we also study the case of the Morse potential as well as the WoodsSaxon potential. This extends our conclusions to these two situations.

\section{Application to the computation of ground states: the nonlinear case}

\subsection{Problem and numerical scheme}

We are interested in computing ground states for nonlinear Schrödinger equations. Most particularly, we consider a nonlinear potential which is the sum of a cubic nonlinearity and a harmonic potential. This kind of nonlinearity arises e.g. in Bose-Einstein condensates [30, 31, 32]. The dimensionless one-dimensional Gross-Pitaevskii equation [33, 34, 35] reads

$$
i \frac{\partial \psi}{\partial t}=-\frac{1}{2} \partial_{x}^{2} \psi+V \psi+\beta|\psi|^{2} \psi, \quad x \in \mathbb{R}
$$

setting $V(x)=\frac{1}{2} x^{2}$ and where the nonlinearity coefficient $\beta$ can be negative or positive. We restrict ourselves to this special nonlinearity but all results can be directly extended to other cases. In view of computing the stationary solutions we write: $\psi(x, t)=e^{-i E t} \phi_{E}(x)$, where 
$E$ is the chemical potential of the condensate and $\phi_{E}$ is a real-valued function independent of time. Let us note that the stability of exactly this kind of problems was studied analytically in $[36,37,38]$ and hence can be checked numerically using our proposed ABCs.

Function $\phi_{E}$ is then solution to

$$
-\alpha \partial_{x}^{2} \phi_{E}+V \phi_{E}+\beta\left|\phi_{E}\right|^{2} \phi_{E}=E \phi_{E}, \quad x \in \mathbb{R},
$$

where $\alpha=\frac{1}{2}$, under the normalization constraint

$$
\left\|\phi_{E}\right\|_{L^{2}(\mathbb{R})}=1 \text {. }
$$

Finally, the function $\phi_{E}$ of the problem (28)-(29) satisfies the boundary conditions $\phi_{E}^{\prime}(0)=0$ and $\phi_{E}( \pm x) \rightarrow 0$ for $x \rightarrow+\infty$. The resulting system is a nonlinear eigenvalue problem under constraint. The eigenfunction $\phi_{E}$ being known, we can determine the associated eigenvalue $E$ by

$$
E=\int_{\mathbb{R}} \alpha\left|\partial_{x} \phi_{E}\right|^{2}+V \phi_{E}^{2}+\beta \phi_{E}^{4} d x
$$

The problem (28)-(29) is solved on a symmetric computational domain $\Omega=]-R ; R$, with $R>0$ and $\Sigma=\{-R ; R\}$. We keep on denoting this domain by $\Omega=]-x_{\ell} ; x_{r}[$. We introduce $\left(E^{0}, \phi^{0}\right)$ as a solution to the boundary value problem with Dirichlet boundary condition

$$
\begin{cases}-\alpha \partial_{x}^{2} \phi_{E}+V \phi_{E}+\beta\left|\phi_{E}\right|^{2} \phi_{E}=E \phi_{E}, & \text { in } \Omega, \\ \phi_{E}=0, & \text { on } \Sigma, \\ \left\|\phi_{E}\right\|_{L^{2}(\Omega)}=1 . & \end{cases}
$$

Analogously, we designate by $\left(E^{M}, \phi^{M}\right)$ the solution computed with a $M$-th order nonlinear ABC obtained from the linear stationary ABCs (6)-(7). To this end, we replace formally the potential $V$ by the new nonlinear potential $V+\beta|\phi|^{2}$ to get the second-order ABC

$$
\partial_{\mathbf{n}} \phi_{E}=\frac{i}{\sqrt{\alpha}} \sqrt{E-V-\beta\left|\phi_{E}\right|^{2}} \phi_{E}, \quad \text { on } \Sigma,
$$

and fourth-order ABC

$$
\partial_{\mathbf{n}} \phi_{E}=\frac{i}{\sqrt{\alpha}} \sqrt{E-V-\beta\left|\phi_{E}\right|^{2}} \phi_{E}+\frac{1}{4} \frac{\partial_{\mathbf{n}}\left(V+\beta\left|\phi_{E}\right|^{2}\right)}{E-V-\beta\left|\phi_{E}\right|^{2}} \phi_{E}, \quad \text { on } \Sigma .
$$

For the sake of clarity, we keep on designating by $\mathrm{SABC}^{M}$ the above $M$-th order $\mathrm{ABC}$.

The interior equation is discretized by the semi-implicit scheme

$$
-\alpha \partial_{x}^{2} \phi^{M, j+1}+V \phi^{M, j+1}+\beta\left|\phi^{M, j}\right|^{2} \phi^{M, j+1}=E^{M, j+1} \phi^{M, j+1},
$$

for $j \geq 0$ and $M=0,2,4$. Now and independently of the boundary condition, the algorithm must be iterative since the interior scheme is nonlinear. As a consequence, we systematically use the fixed point method on the $n$-th eigenvalue $E_{n}^{M}$ and eigenfunction $\phi_{n}^{M}$ for solving the eigenvalue problem. The variational formulation reads

$$
\begin{aligned}
-\alpha\left[\partial_{\mathbf{n}} \phi_{n}^{M, j+1} \psi\right]_{x_{\ell}}^{x_{r}}+\alpha \int_{\Omega} \partial_{x} \phi_{n}^{M, j+1} \partial_{x} \psi d x & +\int_{\Omega} V \phi_{n}^{M, j+1} \psi d x \\
& +\beta \int_{\Omega}\left|\phi_{n}^{M, j}\right|^{2} \phi_{n}^{M, j+1} \psi d x=E_{n}^{M, j+1} \int_{\Omega} \phi_{n}^{M, j+1} \psi d x
\end{aligned}
$$


for any test-function $\psi$. In the Dirichlet case, by choosing $\psi \in H_{0}^{1}(\Omega)$, which makes the first term of the equation vanish, the discrete problem is, for $M=0$,

$$
\left\{\begin{array}{l}
\left(\alpha \mathbb{S}^{0}+\mathbb{M}_{V}^{0}+\beta \mathbb{M}_{\left|\phi_{n}^{M, j}\right|^{2}}^{0}\right) \phi_{n}^{M, j+1}=E_{n}^{M, j+1} \mathbb{M}^{0} \phi_{n}^{M, j+1}, \\
\left\|\mathbb{M}^{0} \boldsymbol{\phi}_{n}^{M, j+1}\right\|_{2}=1
\end{array}\right.
$$

For the ABCs, we use for $\partial_{\mathbf{n}} \phi_{n}^{M, j+1}$ the fixed point version

$$
\partial_{\mathbf{n}} \phi_{n}^{M, j+1}=\frac{i}{\sqrt{\alpha}} \sqrt{E_{n}^{M, j}-V-\beta\left|\phi_{n}^{M, j}\right|^{2}} \phi_{n}^{M, j+1}
$$

for the second-order $\mathrm{ABC}(32)$ and

$$
\partial_{\mathbf{n}} \phi_{n}^{M, j+1}=\left(\frac{i}{\sqrt{\alpha}} \sqrt{E_{n}^{M, j}-V-\beta\left|\phi_{n}^{M, j}\right|^{2}}+\frac{1}{4} \frac{\partial_{\mathbf{n}}\left(V+\beta\left|\phi_{n}^{M, j}\right|^{2}\right)}{E_{n}^{M, j}-V-\beta\left|\phi_{n}^{M, j}\right|^{2}}\right) \phi_{n}^{M, j+1}
$$

for the fourth-order condition (33). Hence, the term $-\alpha\left[\partial_{\mathbf{n}} \phi_{n}^{M, j+1} \varphi\right]_{x_{\ell}}^{x_{r}}$ leads, from a discrete point of view, to a matrix contribution $\mathbb{B}_{M}^{j} \phi^{M, j+1}$ for the $M$-th order ABC, where the matrix coefficients $\mathbb{B}_{M}^{j}$ only depend on the values of $\phi_{n}^{M, j}$ and $E_{n}^{M, j}$. By applying the fixed point algorithm on the $n$-th eigenvalue $E_{n}^{M}$ and eigenvector leads to the iterative scheme $\boldsymbol{\phi}_{n}^{M}$

$$
\left\{\begin{array}{l}
\left(\alpha \mathbb{S}-\alpha \mathbb{B}^{M, j}+\mathbb{M}_{V}+\beta \mathbb{M}_{\left|\phi_{n}^{M, j}\right|^{2}}\right) \phi_{n}^{M, j+1}=E_{n}^{M, j+1} \mathbb{M} \phi_{n}^{M, j+1}, \\
\left\|\mathbb{M} \phi_{n}^{M, j+1}\right\|_{2}=1
\end{array}\right.
$$

The matrix coefficients $\mathbb{B}^{M, j}$ are given by

$$
\left(\mathbb{B}^{M, j}\right)_{1,1}=\frac{i}{\sqrt{\alpha}} \sqrt{E_{n}^{M, j}-V_{\ell}-\beta\left|\phi_{n, \ell}^{M, j}\right|^{2}}+\frac{1}{4} \frac{\partial_{\mathbf{n}}\left(V+\beta\left|\phi_{n}^{M, j}\right|^{2}\right)_{\mid x=x_{\ell}}}{E_{n}^{M, j}-V_{\ell}-\beta\left|\phi_{n, \ell}^{M, j}\right|^{2}}
$$

and

$$
\left(\mathbb{B}^{M, j}\right)_{n_{h}+1, n_{h}+1}=\frac{i}{\sqrt{\alpha}} \sqrt{E_{n}^{M, j}-V_{r}-\beta\left|\boldsymbol{\phi}_{n, r}^{M, j}\right|^{2}}+\frac{1}{4} \frac{\partial_{\mathbf{n}}\left(V+\beta\left|\boldsymbol{\phi}_{n}^{M, j}\right|^{2}\right)_{\mid x=x_{r}}}{E_{n}^{M, j}-V_{r}-\beta\left|\boldsymbol{\phi}_{n, r}^{M, j}\right|^{2}}
$$

for $\mathrm{SABC}^{4}(M=4)$. For $\mathrm{SABC}^{2}(M=2)$, it is sufficient to retain only the first term of each of the above expressions. We have set here: $\phi_{n, \ell}^{M, j}=\phi_{n, \mid x=x_{\ell}}^{M, j}$ and $\phi_{n, r}^{M, j}=\phi_{n, \mid x=x_{r}}^{M, j}$.

As in the linear case, we can formulate the linearized versions of the second- and fourthorder ABCs. These ABCs are then designated by $\mathrm{SABC}_{\text {lin }}^{2,4}$. Doing so, we have the secondorder $\mathrm{ABC}$

$$
\partial_{\mathbf{n}} \phi_{n}^{M, j+1}=-\frac{\sqrt{V}}{\sqrt{\alpha}} \phi_{n}^{M, j+1}-\frac{\beta}{2} \frac{1}{\sqrt{\alpha} \sqrt{V}}\left|\phi_{n}^{M, j}\right|^{2} \phi_{n}^{M, j+1}+\frac{1}{2} \frac{1}{\sqrt{\alpha} \sqrt{V}} E_{n}^{M, j+1} \phi_{n}^{M, j+1}
$$

and the fourth-order $\mathrm{ABC}$

$$
\begin{aligned}
\partial_{\mathbf{n}} \phi_{n}^{M, j+1}= & -\frac{\sqrt{V}}{\sqrt{\alpha}} \phi_{n}^{M, j+1}-\frac{\beta}{2} \frac{1}{\sqrt{\alpha} \sqrt{V}}\left|\phi_{n}^{M, j}\right|^{2} \phi_{n}^{M, j+1}+\frac{1}{2} \frac{1}{\sqrt{\alpha} \sqrt{V}} E_{n}^{M, j+1} \phi_{n}^{M, j+1} \\
& +\left(-\frac{1}{4} \frac{\partial_{\mathbf{n}}\left(V+\beta\left|\phi_{n}^{M, j}\right|^{2}\right)}{V}+\frac{\beta}{4} \frac{\left|\phi_{n}^{M, j}\right|^{2} \partial_{\mathbf{n}}\left(V+\beta\left|\phi_{n}^{M, j}\right|^{2}\right)}{V^{2}}\right) \phi_{n}^{M, j+1} \\
& -E_{n}^{M, j+1} \frac{\partial_{\mathbf{n}}\left(V+\beta\left|\phi_{n}^{M, j}\right|^{2}\right)}{V^{2}} \phi_{n}^{M, j+1} .
\end{aligned}
$$


The iterative scheme then reads

$$
\left\{\begin{array}{l}
\left(\alpha \mathbb{S}-\alpha \mathbb{B}^{M, j}+\mathbb{M}_{V}+\beta \mathbb{M}_{\left|\phi_{n}^{M, j}\right|^{2}}\right) \phi_{n}^{M, j+1}=E_{n}^{M, j+1}\left(\mathbb{M}+\alpha \mathbb{B}_{E, M}^{j}\right) \phi_{n}^{M, j+1} \\
\left\|\mathbb{M} \phi_{n}^{M, j+1}\right\|_{2}=1
\end{array}\right.
$$

The matrix coefficients $\mathbb{B}^{M, j}$ et $\mathbb{B}_{E}^{M, j}$ are given by

$$
\begin{aligned}
\left(\mathbb{B}^{M, j}\right)_{1,1}=-\frac{\sqrt{V_{\ell}}}{\sqrt{\alpha}}-\frac{\beta}{2} \frac{1}{\sqrt{\alpha} \sqrt{V_{\ell}}}\left|\phi_{n, \ell}^{M, j}\right|^{2} & \\
& -\frac{1}{4} \frac{\partial_{\mathbf{n}}\left(V_{\ell}+\beta\left|\phi_{n, \ell}^{M, j}\right|^{2}\right)}{V_{\ell}}+\frac{\beta}{4} \frac{\left|\phi_{n, \ell}^{M, j}\right|^{2} \partial_{\mathbf{n}}\left(V_{\ell}+\beta\left|\phi_{n, \ell}^{M, j}\right|^{2}\right)}{V_{\ell}^{2}}
\end{aligned}
$$

and

$$
\left(\mathbb{B}_{E}^{M, j}\right)_{1,1}=\frac{1}{2} \frac{1}{\sqrt{\alpha} \sqrt{V_{\ell}}}-\frac{\partial_{\mathbf{n}}\left(V_{\ell}+\beta\left|\phi_{n, \ell}^{M, j}\right|^{2}\right)}{V_{\ell}^{2}}
$$

for the fourth-order ABC. The expression of the coefficients of index $\left(n_{h}+1, n_{h}+1\right)$ is the same but taking its value at $x=x_{r}$. We can easily extract the coefficients associated with the second-order ABC by keeping only the first term of each expression. Unlike the linear situation, there is no gain in terms of computational time since the problem is fully nonlinear.

\subsection{Numerical results}

We consider (28) for different values of the parameter $\beta$. For each value, we uniquely determine the fundamental state $n=0$. To get some reference eigenvalues, we numerically compute them on the domain ] - 30; 30[, with a step size $h=10^{-4}$ and $\mathrm{SABC}^{2}(33)$. This method provides some values reported in Table 1 which are conform with the ones given in [34]. Let us note here that we do not give some results for larger values of $\beta$ because the fixed point algorithm then diverges. It would be necessary at this point to use another numerical algorithm (a Newton method or a continuation method) for solving the problem with an ABC. Finally, we present in the sequel the absolute errors: $\Delta E=\left|E^{\text {num }}-E^{\text {ref }}\right|$ and $\Delta \phi(0)=\left|\phi^{\text {num }}(0)-\phi^{\text {ref }}(0)\right|$, where 'ref' refers to the values in Table 1 and 'num' to the ones computed with the proposed method.

\begin{tabular}{ccc}
$\beta$ & $\phi_{E}(0)$ & $E$ \\
\hline-6.2742 & 1.265512713848083 & -4.956873352670034 \\
-2.5097 & 0.913230941756339 & -0.806257128073956 \\
3.1371 & 0.645961493829006 & 1.526594842533555
\end{tabular}

Table 1: Numerical values $E^{\mathrm{ref}}$ and $\phi_{E}^{\mathrm{ref}}(0)$ computed on a larger domain for different $\beta$.

For the simulations, the initialization of the fixed point algorithm uses the exact harmonic potential solution $(\beta=0): \phi_{0}^{M, 0}(x)=\frac{1}{\pi^{1 / 4}} e^{-x^{2} / 2}$. The fixed point algorithm tolerance is $\varepsilon=10^{-12}$ and the mesh size of the linear finite element method is $h=10^{-3}$. Figures 11,12 and 13 report the error on both the eigenvalue and eigenfunction at the origin depending on the right endpoint $x_{r}$, for the values $\beta=-6.2742, \beta=-2.5097$ and $\beta=3.1371$, respectively.

Generally speaking, for a given case, all the algorithms converge with about the same number of iterations, independently of the boundary condition. We also note that, for 
negative values of $\beta$, the linearized ABCs lead to the same accuracy as the nonlinear ABCs (not reported here) for a similar computational time. In Figures 11 and 12, we only present the results for the Dirichlet boundary condition and $\mathrm{SABC}^{2,4}$. For $\beta>0$ (Figure 13), the linearized $\mathrm{ABC}$ possess an accuracy at least equal to the one with $\mathrm{SABC}^{2,4}$. We do not have any explanation about this fact. For $\beta=-6.2742$ (Figure 11), all the algorithms converge in 23 iterations. The ABCs improve the accuracy from a factor 10 compared with the Dirichlet boundary condition for $x_{r}=1.5$, and almost 100 when $x_{r}=2$, then for $x_{r} \geq 2.5$, all the boundary conditions have the same accuracy: $10^{-5}$. The precision of the secondorder ABC is slightly better than the fourth-order ABC. There is no explanation here about this behavior but we recall that $\mathrm{ABC}$ for the nonlinear case where obtained by the formal argument: "the potential is replaced by the nonlinearity". For $\beta=-2.5097$ (Figure 12), the convergence takes 14 iterations. The ABCs again provides a gain of precision compared with the Dirichlet boundary condition for $x_{r}$ between 1.5 and 3.5, with a better accuracy for the second-order ABC (see the points $x_{r}=3$ and $x_{r}=2.5$ for example). For $\beta=3.13712$, the situation is quite similar but requires 77 iterations to converge. Unlike, the two previous cases, the linearized ABCs give a slightly better accuracy than for the original ABCs.

\section{Extension to $N$-dimensional stationary problems}

In the $N$-dimensional linear time-dependent case, the ABCs take the following form

$$
\mathrm{ABC}_{2}^{1}: \quad \partial_{\mathbf{n}} u-i \mathrm{Op}\left(\sqrt{-\tau+\Delta_{\Sigma}+\widetilde{V}}\right) u=0
$$

for the first-order condition and

$$
\begin{aligned}
\mathrm{ABC}_{2}^{2}: \quad \partial_{\mathbf{n}} u- & i \mathrm{Op}\left(\sqrt{-\tau+\Delta_{\Sigma}+\widetilde{V}}\right) u \\
& +\mathcal{H O p}\left(\left(-\tau+\Delta_{\Sigma}+\widetilde{V}\right)^{-1}\right)\left(i \partial_{t}+\widetilde{V}\right) u=0
\end{aligned}
$$

for the second-order one on $\Sigma \times \mathbb{R}^{+}$. The Schrödinger equation under consideration is the following

$$
\begin{cases}i \partial_{t} u+\Delta u+\widetilde{V}(\mathbf{x}) u=0, & \forall(\mathbf{x}, t) \in \mathbb{R}^{N} \times \mathbb{R}^{+}, \\ u(\mathbf{x}, 0)=u_{0}(\mathbf{x}), & \mathbf{x} \in \mathbb{R}^{N},\end{cases}
$$

Here, the computational domain $\Omega$ is a bounded set of the $\mathbb{R}^{N}$ dimensional space. Its boundary $\Sigma$ is supposed to be a $(N-1)$-dimensional convex and compact manifold. Its mean curvature $\mathcal{H}$ is defined by: $\mathcal{H}=\operatorname{trace}(\mathcal{R}) /(N-1)$, where $\mathcal{R}$ is the curvature tensor of the surface. For example, for a two-dimensional surface, we have: $\mathcal{H}=\kappa / 2$, where $\kappa$ is the local curvature at a point of the surface. For a 2 -sphere of radius $R$, we get $\mathcal{H}=1 / R$. The operator $\Delta_{\Sigma}$ is the Laplace-Beltrami operator over the surface. For the sphere it is defined by

$$
\Delta_{\Sigma} f:=\frac{1}{r^{2} \sin \varphi} \frac{\partial}{\partial \varphi}\left(\sin \varphi \frac{\partial f}{\partial \varphi}\right)+\frac{1}{r^{2} \sin ^{2} \varphi} \frac{\partial^{2} f}{\partial \theta^{2}} .
$$

for a function $f$ expressed in spherical coordinates $(r, \varphi, \theta)$. Other expressions exist for the hypersphere and other manifolds. The asymptotics behind the construction of the ABCs is somewhat different which results in a different definition of the order of an ABC. We do not develop the whole theory and refer to [29] for more details in the 2-dimensional case. 
To get the ABCs for the $N$-dimensional stationary case

$$
-\alpha \Delta u+V(\mathbf{x}) u=E u, \quad \text { in } \Omega,
$$

we make the substitutions: $-\tau \rightarrow E / \alpha$ and $\widetilde{V} \rightarrow-V / \alpha$. This leads to the first-order Stationary ABC:

$$
\mathrm{SABC}^{1}: \partial_{\mathbf{n}} u+\frac{1}{\sqrt{\alpha}} \sqrt{V-\left(\alpha \Delta_{\Sigma}+E\right)} u=0
$$

and

$$
\mathrm{SABC}^{2}: \partial_{\mathbf{n}} u+\frac{1}{\sqrt{\alpha}} \sqrt{V-\left(\alpha \Delta_{\Sigma}+E\right)} u+\mathcal{H}(V-E)\left(V-\left(\alpha \Delta_{\Sigma}+E\right)\right)^{-1} u=0
$$

As in the one-dimensional case, both conditions are nonlinear with respect to the energy $E$. Furthermore, the square-root involves now the surface Laplace-Beltrami operator $\Delta_{\Sigma}$. Here, we propose the formal asymptotics: $V \gg \alpha \Delta_{\Sigma}+E$ which can be justified by theoretical arguments of operator theory [29]. Using a second-order Taylor expansion, we obtain the approximate linearized SABC

$$
\mathrm{SABC}_{\text {lin }}^{1}: \partial_{\mathbf{n}} \tilde{\phi}_{E}+\frac{\sqrt{V}}{\sqrt{\alpha}} \tilde{\phi}_{E}-\frac{\sqrt{\alpha}}{2 \sqrt{V}} \Delta_{\Sigma} \tilde{\phi}_{E}+\frac{\sqrt{\alpha}}{2 \sqrt{V}} E \tilde{\phi}_{E}=0
$$

on $\Sigma$. For the second-order SABC, we do not really have to linearize the corrective term which can be considered linearly through the introduction of an auxiliary function $\Psi_{E}$. More precisely, we have

$$
\mathrm{SABC}_{\text {lin }}^{2}: \partial_{\mathbf{n}} \tilde{\phi}_{E}+\frac{\sqrt{V}}{\sqrt{\alpha}} \tilde{\phi}_{E}-\frac{\sqrt{\alpha}}{2 \sqrt{V}} \Delta_{\Sigma} \tilde{\phi}_{E}+\frac{\sqrt{\alpha}}{2 \sqrt{V}} E \tilde{\phi}_{E}+\mathcal{H}(V-E) \Psi_{E}=0
$$

coupled to the surface equation: $-\alpha \Delta_{\Sigma} \Psi_{E}+(V-E) \Psi_{E}-\tilde{\phi}_{E}=0$. With such a trick, the coupled system with unknowns $\left(\tilde{\phi}_{E}, \Psi_{E}\right)$ remains linear and is well-adapted to a symmetrical weak formulation for instance. Moreover, each term can be very easily implemented in usual numerical codes based for example on finite difference, finite element or spectral methods. The adaptation to the nonlinear stationary Schrödinger equation (for a smooth nonlinearity like $\left.f(u)=\beta|u|^{2}\right):-\alpha \Delta u+V(\mathbf{x}) u+f(u) u=E u$, in $\Omega$, can be made by simply replacing $V(\mathbf{x})$ by $V(\mathbf{x})+f(u)$ in the above ABCs.

\section{Conclusion}

We have proposed some accurate and physically admissible absorbing boundary conditions for modeling linear and nonlinear stationary Schrödinger equations with variable potentials. Based on numerical schemes, these boundary conditions have been validated for many configurations including linear and nonlinear ground-state computations. Furthermore, the extension to $N$-dimensional problems that can be used in scattering problems like $[39$, Section 12.1], is given.

Future extensions would include variable mass Schrödinger equations [40] among others. It might also be valuable to extent the presented work to systems of Schrödinger equations that arise as so-called multiband effective mass approximations (MEMAs) to model electronic states in modern semiconductor nanostructures, cf. [41, 42, 43]. Let us finally remark that applications to generalized Schrödinger equations could also be developed by adapting the methods developed in $[2,44]$. 


\section{References}

[1] X. Antoine, A. Arnold, C. Besse, M. Ehrhardt, A. Schädle, A review of transparent and artificial boundary conditions techniques for linear and nonlinear Schrödinger equations, Commun. Comput. Phys. 4 (4) (2008) 729-796.

[2] X. Antoine, C. Besse, P. Klein, Absorbing boundary conditions for the one-dimensional Schrödinger equation with an exterior repulsive potential, J. Comput. Phys. 228 (2) (2009) 312-335.

[3] X. Antoine, C. Besse, M. Ehrhardt, P. Klein, Modeling boundary conditions for solving stationary Schrödinger equations, Report Preprint 10/04 (http://www.math.uniwuppertal.de), Lehrstuhl für Angewandte Mathematik und Numerische Mathematik.

[4] W. Bao, I.-L. Chern, F. Y. Lim, Efficient and spectrally accurate numerical methods for computing ground and first excited states in Bose-Einstein condensates, J. Comput. Phys. 219 (2) (2006) 836-854. doi:10.1016/j.jcp.2006.04.019.

[5] W. Bao, Y. Cai, H. Wang, Efficient numerical methods for computing ground states and dynamics of dipolar Bose-Einstein condensates, J. Comput. Phys. 229 (20) (2010) 7874-7892. doi:10.1016/j.jcp.2010.07.001.

[6] W. Bao, H. Li, J. Shen, A Generalized-Laguerre-Fourier-Hermite pseudospectral method for computing the dynamics of rotating Bose-Einstein condensates, SIAM J. Sci. Comput. 31 (5) (2009) 3685-3711. doi:10.1137/080739811.

[7] W. Bao, J. Shen, A generalized-Laguerre-Hermite pseudospectral method for computing symmetric and central vortex states in Bose-Einstein condensates, Journal of Computational Physics 227 (23) (2008) 9778-9793. doi:10.1016/j.jcp.2008.07.017.

[8] V. Ledoux, M. Van Daele, G. Vanden Berghe, MATSLISE: A MATLAB package for the numerical solution of Sturm-Liouville and Schrodinger equations, ACM Trans. Math. Softw. 31 (4) (2005) 532-554.

[9] V. Ledoux, L. G. Ixaru, M. Rizea, M. Van Daele, G. Vanden Berghe, Solution of the Schrodinger equation over an infinite integration interval by perturbation methods, revisited, Comput. Phys. Commun. 175 (9) (2006) 612-619. doi:10.1016/j.cpc.2006.07.006.

[10] V. Ledoux, Study of Study of Special Algorithms for solving Sturm-Liouville and Schrodinger Equations, Ph.D. Thesis, Dept. of Applied Mathematics and Computer Science, Ghent University, 2007.

[11] N. Ben Abdallah, P. Degond, P. A. Markowich, On a one-dimensional SchrödingerPoisson scattering model, Z. Angew. Math. Phys. 48 (1) (1997) 135-155.

[12] A. Arnold, Mathematical concepts of open quantum boundary conditions, Trans. Theory Stat. Phys. 30 (2001) 561-584.

[13] C. Lent, D. Kirkner, The quantum transmitting boundary method, J. Appl. Phys. 67 (10) (1990) 6353-6359. 
[14] D. Kirkner, C. Lent, S. Sivaprakasam, The numerical simulation of electron transmission through a two-dimensional quantum device by the finite element method, Int. J. Numer. Meth. Engineering. 29 (1990) 1527-1537.

[15] M. Heinen, H.-J. Kull, Radiation boundary conditions for the numerical solution of the three-dimensional time-dependent Schrödinger equation with a localized interaction, Physical Review E 79 (5).

[16] M. Heinen, H.-J. Kull, Numerical calculation of strong-field laser-atom interaction: An approach with perfect reflection-free radiation boundary conditions, Laser Physics 20 (3) (2010) 581-590.

[17] M. Ehrhardt, R. E. Mickens, Solutions to the discrete Airy equation: Application to parabolic equation calculations, J. Comput. Appl. Math. 172 (1) (2004) 183-206.

[18] M. Ehrhardt, A. Zisowsky, Fast calculation of energy and mass preserving solutions of Schrödinger-Poisson systems on unbounded domains, J. Comput. Appl. Math. 187 (1) (2006) 1-28.

[19] C. Moyer, Numerov extension of transparent boundary conditions for the Schrödinger equation in one dimension, Amer. J. Phys. 72 (3) (2004) 351-358.

[20] C. Moyer, Numerical solution of the stationary state Schrödinger equation using transparent boundary conditions, Comput. Sci. Engrg. 8 (4) (2006) 32-40.

[21] M. Ehrhardt, C. Zheng, Exact artificial boundary conditions for problems with periodic structures, J. Comput. Phys. 227 (14) (2008) 6877-6894.

[22] L. Burgnies, Mécanismes de conduction en régime ballistique dans les dispositifs électroniques quantiques, Ph.D. thesis, Université des Sciences et Technologies de Lille (1997).

[23] W. Frensley, Boundary conditions for open quantum driven far from equilibrium, Rev. Mod. Phys. 62 (1990) 745-791.

[24] P. Markowich, C. Ringhofer, C. Schmeiser, Semiconductor Equations, Springer, NewYork, 1990.

[25] C. Schmeiser, Computational methods for semiclassical and quantum transport in semiconductor devices, Acta Numerica 3 (1997) 485-521.

[26] M. Taylor, Pseudodifferential Operators, Vol. 34 of Princeton Mathematical Series, Princeton University Press, Princeton, N.J., 1981.

[27] J. Chazarain, A. Piriou, Introduction to the Theory of Linear Partial Differential Equations, North-Holland, Amsterdam/New-York, 1982.

[28] H. Shao, Z. Wang, Arbitrarily precise numerical solutions of the one-dimensional Schrödinger equation, Comput. Phys. Commun. 180 (1) (2009) 1-7.

[29] P. Klein, Construction et analyse de conditions aux limites artificielles pour des équations de Schrödinger avec potentiels et non linéarités, Ph.D. thesis, Nancy Université, France (http://www.iecn.u-nancy.fr/ klein/These_PK_hr.pdf) (2010). 
[30] E. Gross, Structure of a quantized vortex in boson systems, Nuovo Cimento 20 (3) (1961) 454-477.

[31] L. Pitaevskii, Vortex lines in an imperfect Bose gas, Soviet Physics JETP 13 (2) (1961) $451-454$.

[32] W. Bao, The nonlinear Schrödinger equation and applications in Bose-Einstein condensation and plasma physics, in: Dynamics in Models of Coarsening, Condensation and Quantization, Vol. 9 of IMS Lecture Notes Series, World Scientific, 2007, pp. 215-255.

[33] W. Bao, Q. Du, Computing the ground state solution of Bose-Einstein condensates by a normalized gradient flow, SIAM J. Sci. Comput. 25 (5) (2004) 1674-1697.

[34] W. Bao, W. Tang, Ground-state solution of Bose-Einstein condensate by directly minimizing the energy functional, J. Comput. Phys. 187 (1) (2003) 230-254.

[35] M. Dehghan, A. Taleei, A compact split-step finite difference method for solving the nonlinear Schrödinger equations with constant and variable coefficients, Comput. Phys. Commun. 181 (2010) 43-51.

[36] R. Thelwell, J. Carter, B. Deconinck, Instabilities of one-dimensional stationary solutions of the cubic nonlinear Schrödinger equation, J. Phys. A 39 (2006) 73-84.

[37] L. Carr, C. Clark, W. Reinhardt, Stationary solutions of the one-dimensional nonlinear Schrödinger equation. I. Case of repulsive nonlinearity, Phys. Rev. A 62 (2000) 063610.

[38] L. Carr, C. Clark, W. Reinhardt, Stationary solutions of the one-dimensional nonlinear Schrödinger equation. II. Case of attractive nonlinearity, Phys. Rev. A 62 (2000) 063611.

[39] B. Bransden, C. Joachain, Physics of Atoms and Molecules, Prentice Hall (Pearson Education Ltd, Harlow, England), 2003.

[40] M. Betcke, H. Voss, Analysis and efficient solution of stationary Schrödinger equation governing electronic states of quantum dots and rings in magnetic feld, Report 143, TU Hamburg-Harburg (2010) submitted to J. Comput. Phys.

[41] A. Zisowsky, A. Arnold, M. Ehrhardt, T. Koprucki, Discrete transparent boundary conditions for transient $k \cdot p$-Schrödinger equations with application to quantumheterostructures, J. Appl. Math. Mech. 85 (11) (2005) 793-805.

[42] D. Klindworth, Discrete transparent boundary conditions for multiband effective mass approximations, Master's thesis, Technische Universität Berlin (2009).

[43] S. Odermatt, M. Luisier, B. Witzigmann, Bandstructure calculation using the $k \cdot p$ method for arbitrary potentials with open boundary conditions, J. Appl. Phys. 97 (2005) 046104.

[44] X. Antoine, C. Besse, V. Mouysset, Numerical schemes for the simulation of the two-dimensional Schrödinger equation using non-reflecting boundary conditions, Math. Comp. 73 (248) (2004) 1779-1799. 


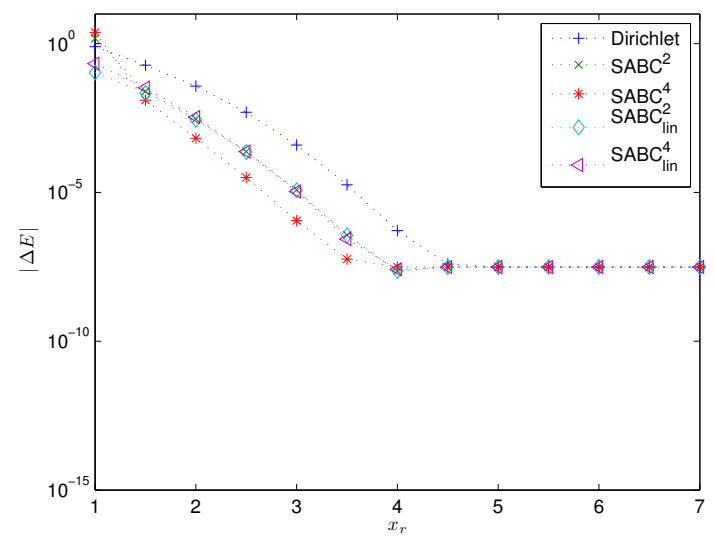

(a) Eigenvalue

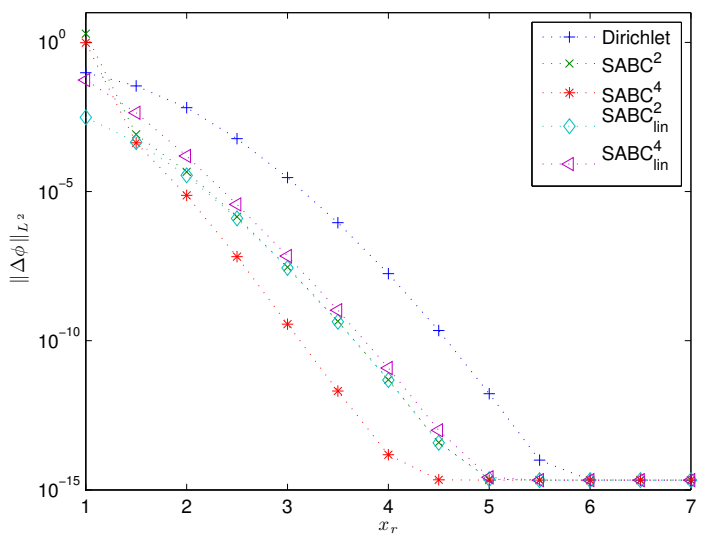

(b) Eigenvector

Figure 1: Example 1: Error $(n=0)$.

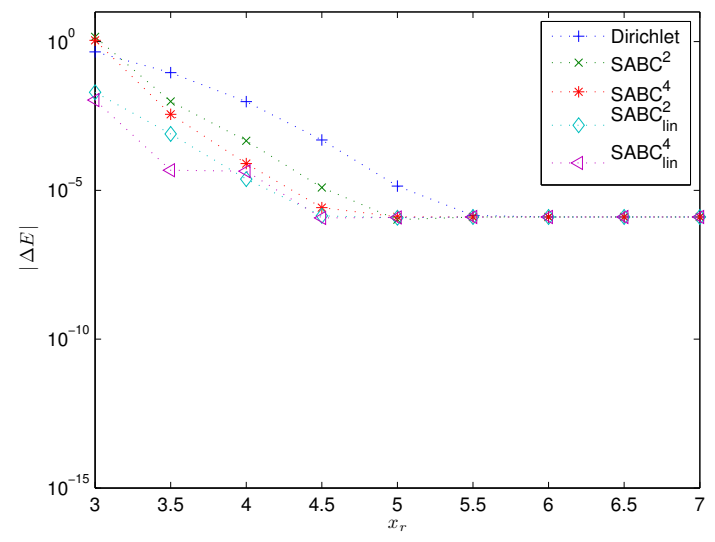

(a) Eigenvalue

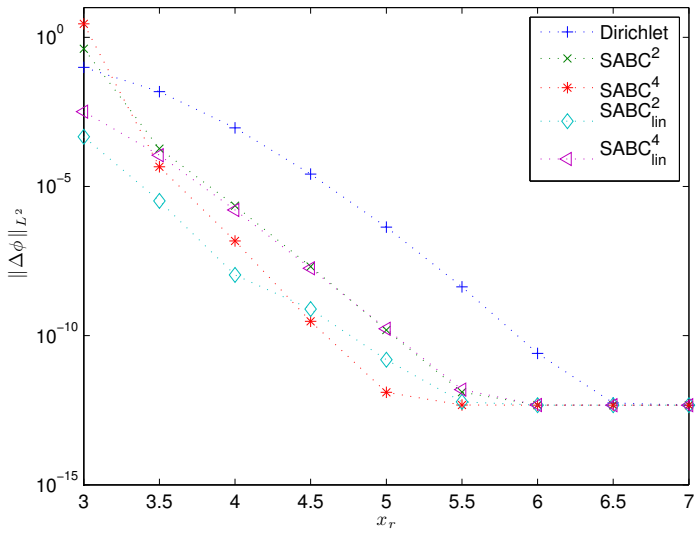

(b) Eigenvector

Figure 2: Example 1: Error $(n=4)$.

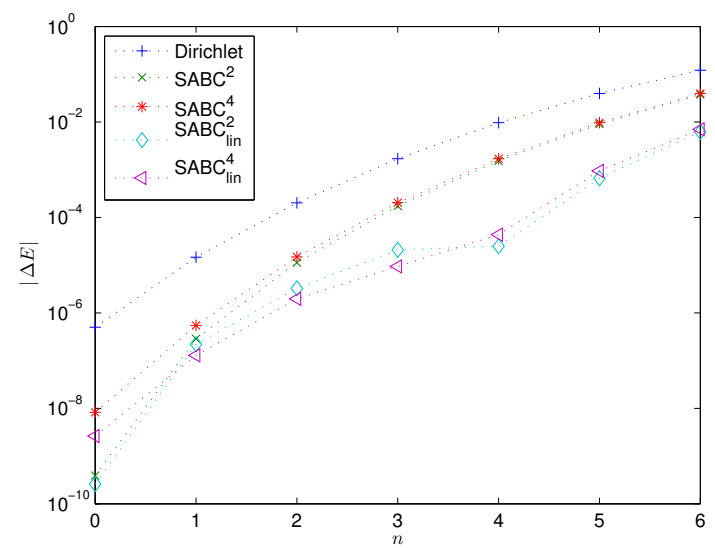

(a) Direct approach

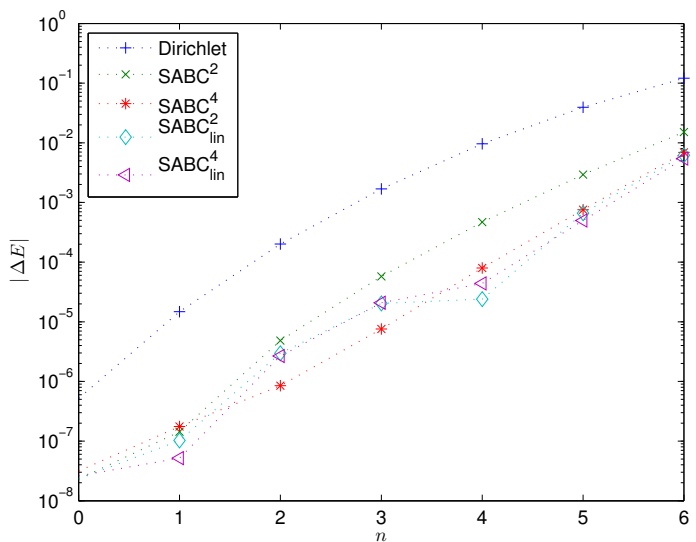

(b) Loop approach

Figure 3: Example 1: Error on the eigenvalue depending on $n(\Omega=]-4 ; 4[)$. 


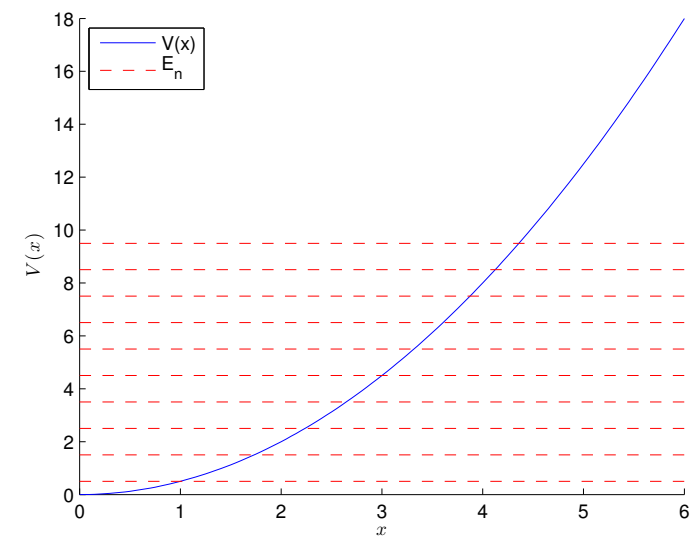

Figure 4: Example 1: Harmonic potential and the energies $E_{n}$ for $0 \leq n \leq 9$.

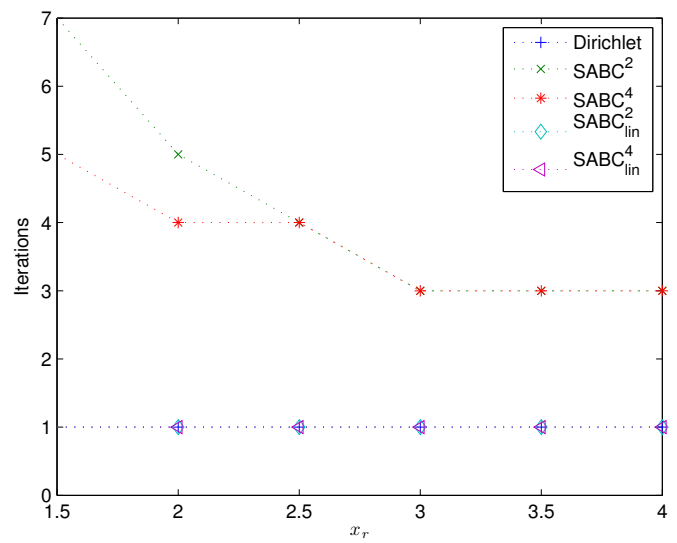

(a) $n=0$

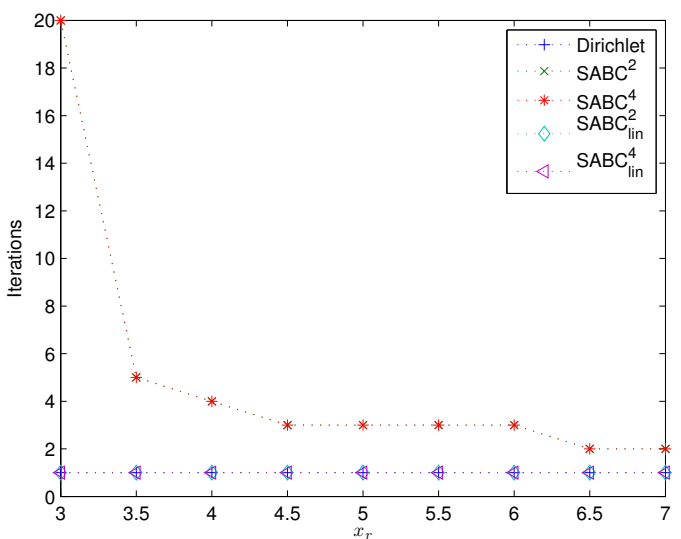

(b) $n=4$

Figure 5: Example 1: Number of iterations needed for the algorithms associated with the different ABCs, with respect to $x_{r}$ for $n=0$ and $n=4$.

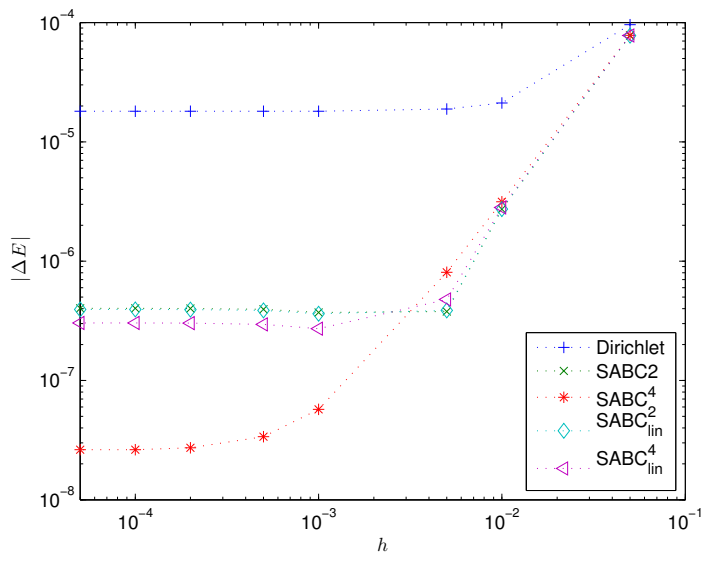

(a) Eigenvalue

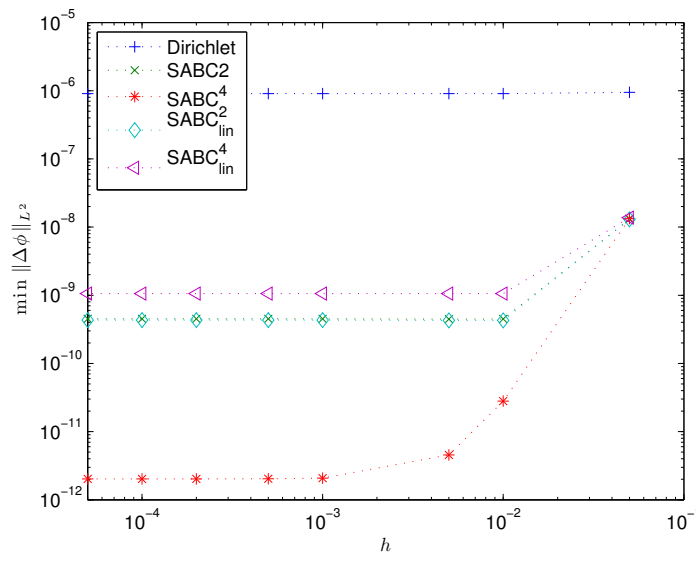

(b) Eigenfunction

Figure 6: Example 1: Error $\left(x_{r}=3.5\right.$ and $\left.n=0\right)$ with respect to $h$. 


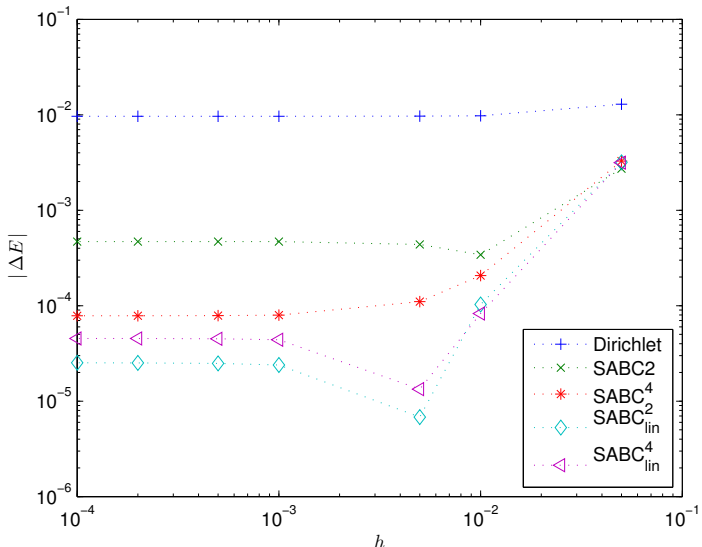

(a) Eigenvalue

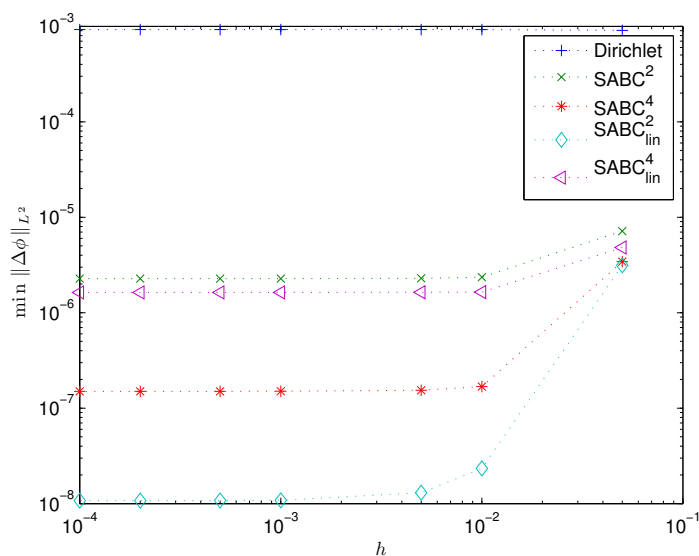

(b) Eigenfunction

Figure 7: Example 1: Error $\left(x_{r}=4\right.$ and $\left.n=4\right)$ with respect to $h$.

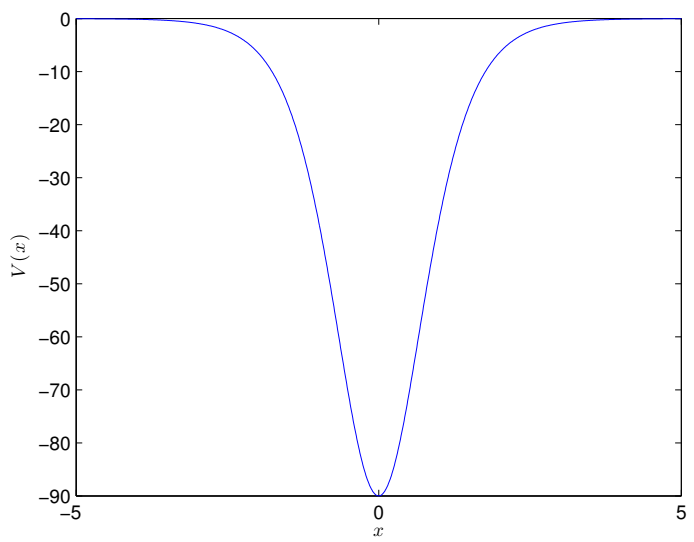

(a) Pöschl-Teller potential

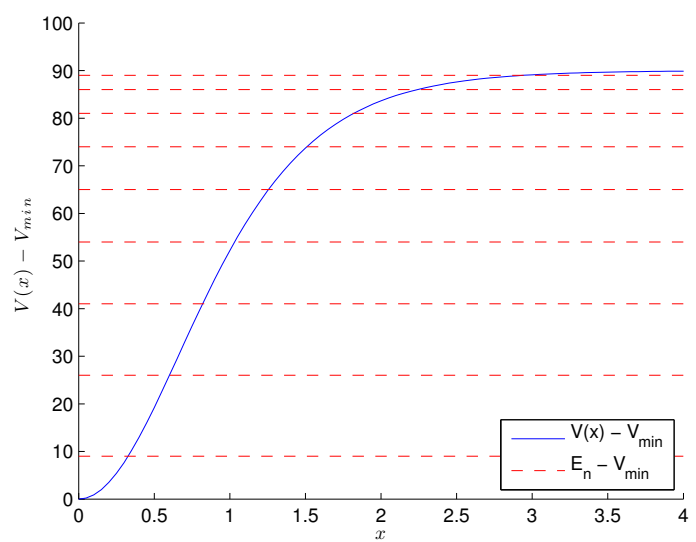

(b) Energy levels

Figure 8: Example 2: Pöschl-Teller potential and its first nine energy levels. 


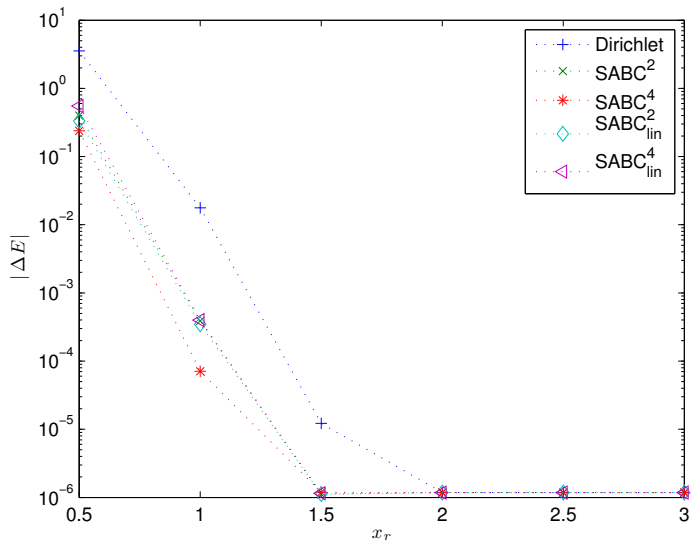

(a) $n=0$

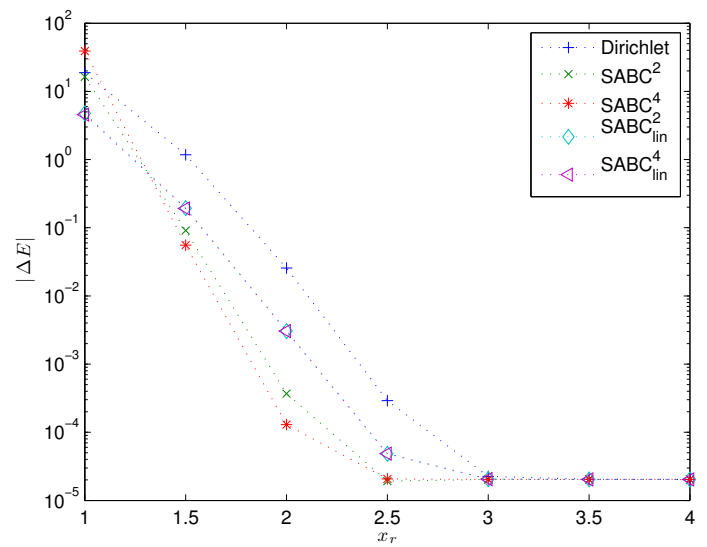

(b) $n=4$

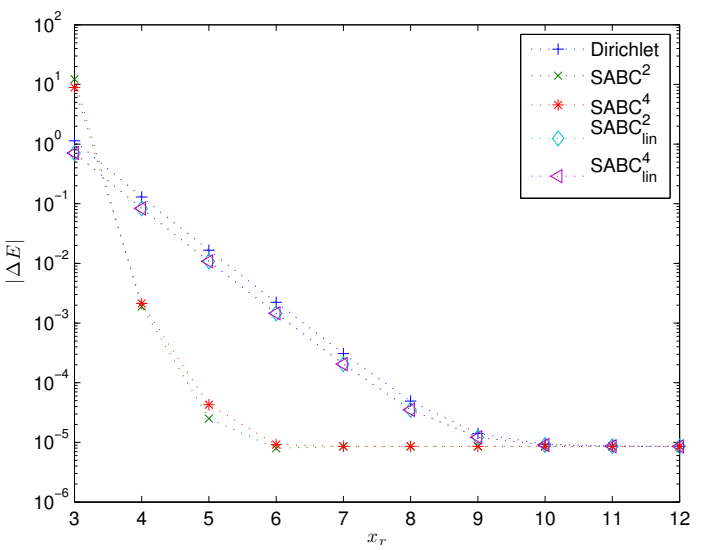

(c) $n=8$

Figure 9: Example 2: Error of the eigenvalues for the different ABCs and the Pöschl-Teller potential. 


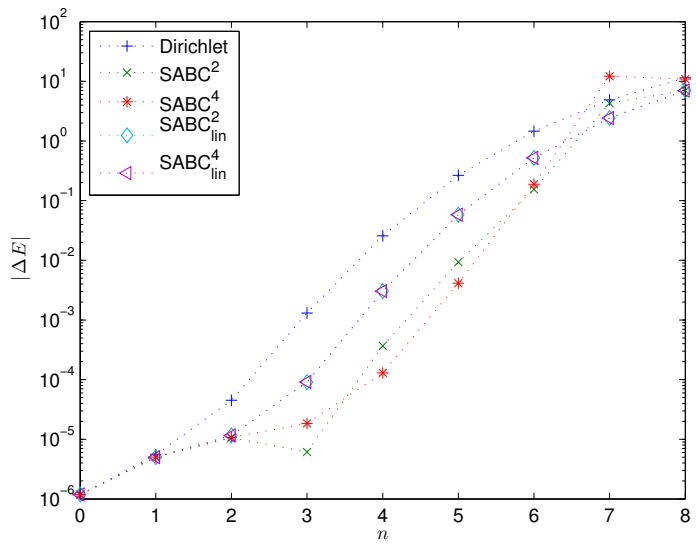

(a) $x_{r}=2$ (loop)

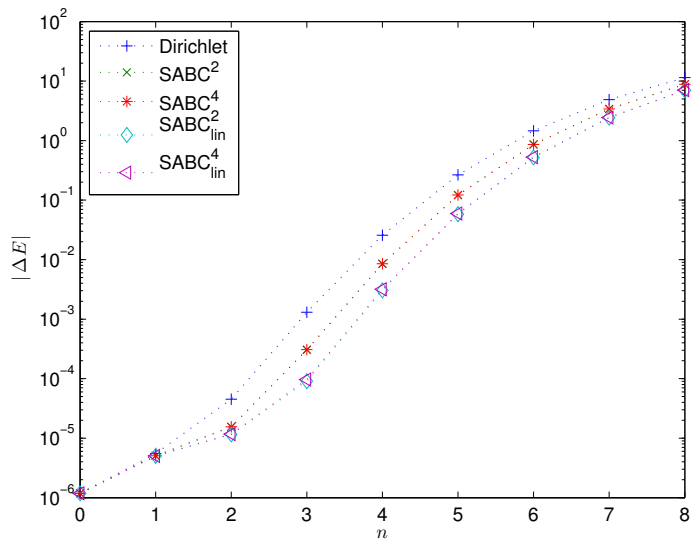

(c) $x_{r}=2$ (direct)

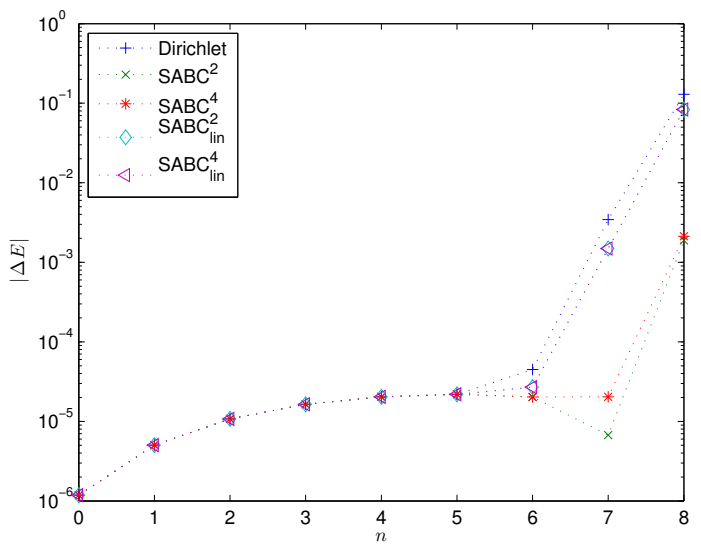

(b) $x_{r}=4$ (loop)

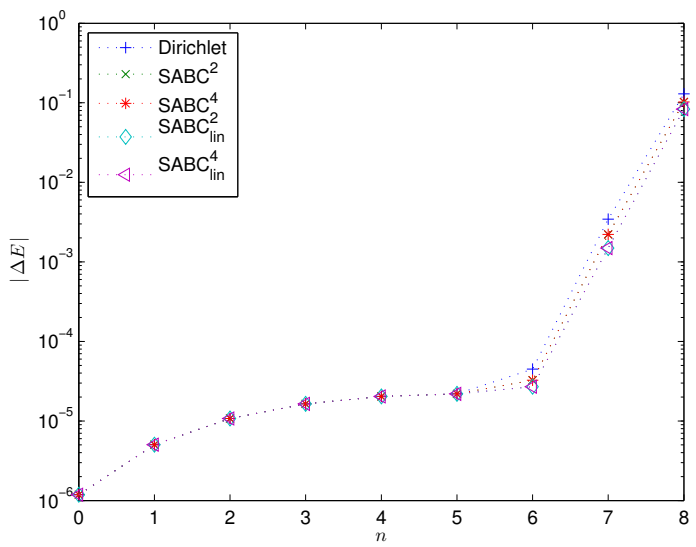

(d) $x_{r}=4$ (direct)

Figure 10: Example 2: Error on the eigenvalues according to $n$ for the Pöschl-Teller potential.

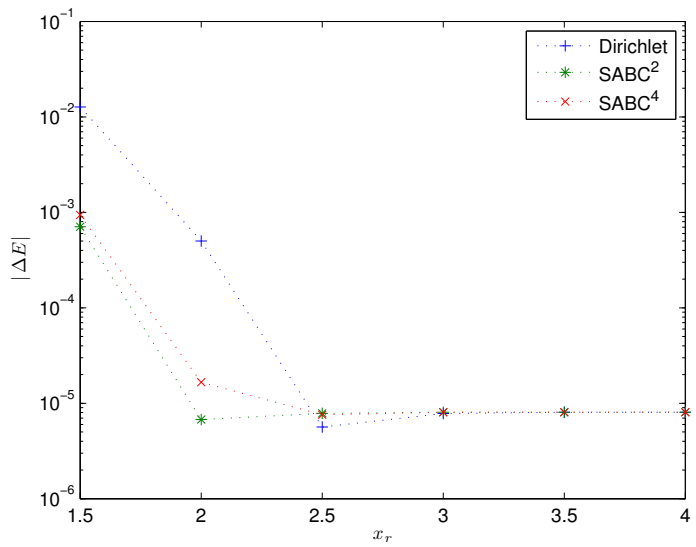

(a) Eigenvalue

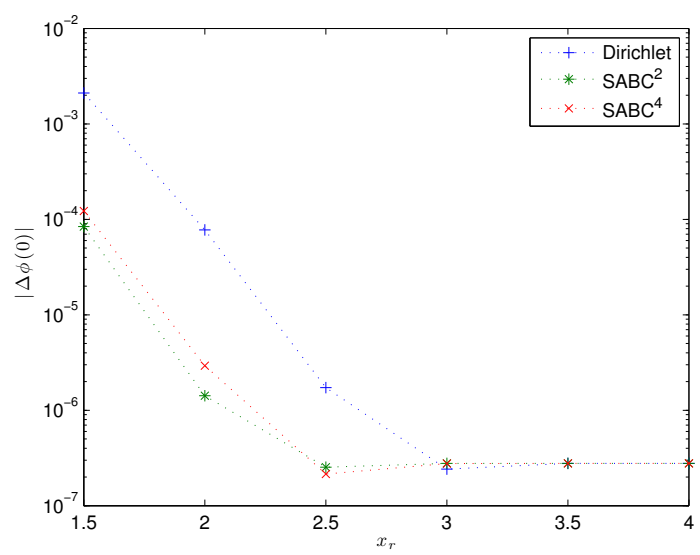

(b) Value of the eigenfunction at the origin

Figure 11: Errors $\Delta E$ and $\Delta \phi(0)$ for $\beta=-6.2742$. 


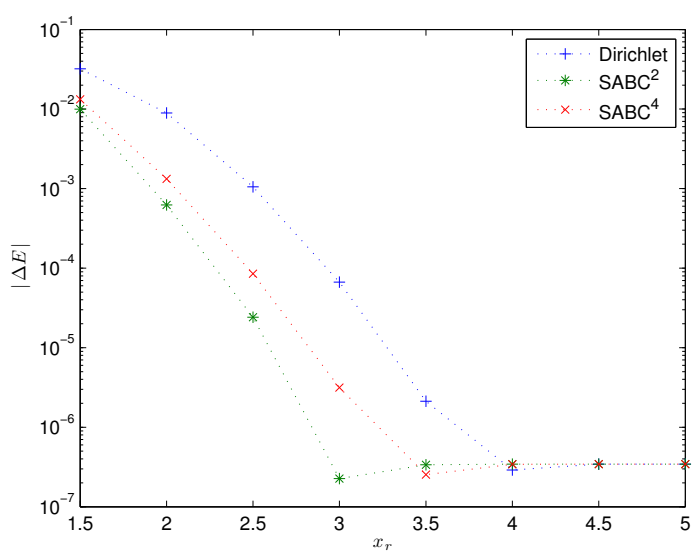

(a) Eigenvalue

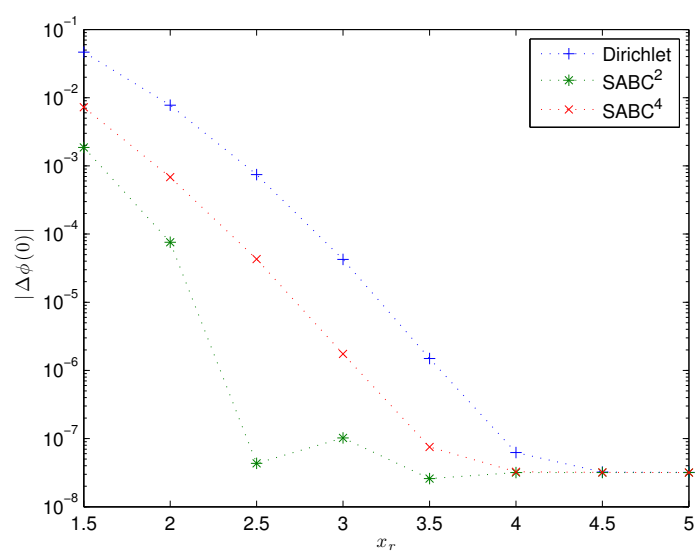

(b) Value of the eigenfunction at the origin

Figure 12: Errors $\Delta E$ and $\Delta \phi(0)$ for $\beta=-2.5097$.

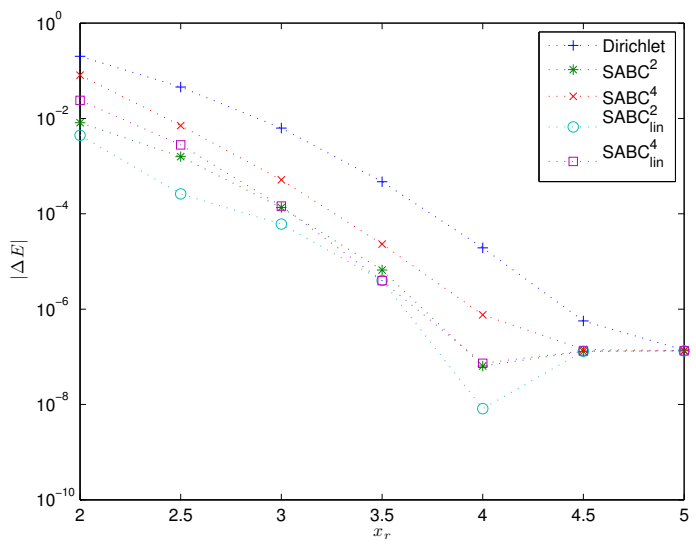

(a) Eigenvalue

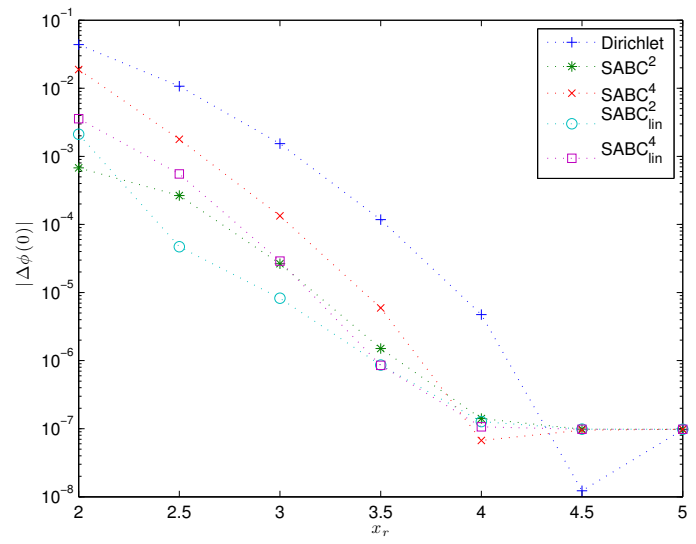

(b) Value of the eigenfunction at the origin

Figure 13: Errors $\Delta E$ and $\Delta \phi(0)$ for $\beta=3.1371$. 